\title{
Treatment of Nightmares Using Imagery Rehearsal Therapy: A Multiple Baseline Study
}

\author{
Michael R. Nadorff \\ West Virginia University
}

Follow this and additional works at: https://researchrepository.wvu.edu/etd

\section{Recommended Citation}

Nadorff, Michael R., "Treatment of Nightmares Using Imagery Rehearsal Therapy: A Multiple Baseline Study" (2012). Graduate Theses, Dissertations, and Problem Reports. 4902.

https://researchrepository.wvu.edu/etd/4902

This Dissertation is protected by copyright and/or related rights. It has been brought to you by the The Research Repository @ WVU with permission from the rights-holder(s). You are free to use this Dissertation in any way that is permitted by the copyright and related rights legislation that applies to your use. For other uses you must obtain permission from the rights-holder(s) directly, unless additional rights are indicated by a Creative Commons license in the record and/ or on the work itself. This Dissertation has been accepted for inclusion in WVU Graduate Theses, Dissertations, and Problem Reports collection by an authorized administrator of The Research Repository @ WVU.

For more information, please contact researchrepository@mail.wvu.edu. 
Treatment of Nightmares Using Imagery Rehearsal Therapy: A Multiple Baseline Study

\author{
Michael R. Nadorff
}

Dissertation submitted to the Eberly College of Arts and Sciences at the West Virginia University in Partial Fulfillment of the Requirements for the degree of

\author{
Doctor of Philosophy \\ In \\ Psychology
}

\begin{abstract}
Amy Fiske, Ph.D., C.B.S.M., Chair William Fremouw, Ph.D., A.B.P.P. Hawley Montgomery-Downs, Ph.D. Claire St. Peter, Ph.D., BCBA-D Laurie Theeke, Ph.D., R.N., C.F.N.P.
\end{abstract}

\author{
Department of Psychology \\ Morgantown, West Virginia
}

2012

Keywords: Imagery Rehearsal Therapy; Nightmare Treatment; Nightmares; Sleep Disorders Copyright 2012 Michael R. Nadorff 


\begin{abstract}
Treatment of Nightmares Using Imagery Rehearsal Therapy: A Multiple Baseline Study Michael R. Nadorff
\end{abstract}

Nightmares have been linked with several negative consequences, including death by suicide. Further, nightmare sufferers report that nightmares have a significant negative impact on their lives. To address this problem, several nightmare treatments have been studied, with Imagery Rehearsal Therapy (IRT) having the greatest amount of empirical support. However, there are many unanswered questions in regard to IRT, such as how quickly one may see an effect and whether or not there is a temporary increase in nightmares prior to a decrease. The current study aimed to address these questions by examining IRT using a non-concurrent multiple baseline design. Four participants took part in the study, with each receiving IRT over the course of one session. IRT did not lead to a substantial decrease in nightmare frequency or severity in this sample, despite strong treatment adherence. The lack of a finding may be due to there being a great deal of intra-individual variability in frequency of nightmares. . There was no substantial increase in nightmare frequency, severity, or negative affect in the week following treatment. However, it is possible that the treatment was ineffective, which could explain why no temporary increase in nightmares or negative affect was observed. Implications and future directions are discussed. 


\section{Acknowledgments}

There are many individuals who deserve acknowledgement for their assistance with this project. When I began this project more than a year ago, I had never conducted a treatment study, used a multiple baseline design, conducted a qualitative study, or utilized actigraphy for research purposes. It would not be inaccurate to say that the project was more novel than familiar for me. When I look back, it reminds me of Isaac Newton's quote "If I have seen a little further it is by standing on the shoulders of giants.” Although Newton was referring to the researchers that preceded him, I feel the same way about those who helped me. Truly, without them this project would have been impossible.

I am deeply grateful to Dr. St. Peter, who helped me at every stage of the project, from design through data analysis. Her assistance not only made the project possible, but taught me a great deal about single-subject research. Similarly, Dr. Montgomery-Downs provided the actigraphs used in the project, but more importantly, taught me how to utilize them for research. This research would not have been possible without Dr. Fremouw, who provided valuable consultation on conducting clinical research and enabled me to use the department clinic to see participants. Lastly, Dr. Theeke and her colleague, Dr. Smith, provided me with guidance that enabled me to complete the qualitative portion of the study. All of these contributions helped make the current project possible, but more importantly, taught me a great deal about research, and will undoubtedly influence my future work. As such, I cannot thank these individuals enough for their guidance and assistance.

I would also like to acknowledge my advisor and mentor, Dr. Fiske. I cannot quantify how much of my training I owe to her, but I do know that not a day goes by that I do not use something she taught me. She provided me with enough support to grow and be successful, but 
also enough freedom to follow my interests and develop a research program that is uniquely mine. I am not sure what more you can ask from your advisor. I hope I will be able to be as strong, yet flexible, an advisor to my students as Dr. Fiske was for me.

Last, but certainly not least, I would like to thank my loving wife, Danielle. Having survived a dissertation herself, she is extremely understanding of the stress caused by a dissertation. She knew when I needed support, when I needed space, and when to intervene in order to give me a break from my work. Without a doubt she helped me keep my sanity through this difficult and trying process, and I can never thank her enough. 


\section{Table of Contents}

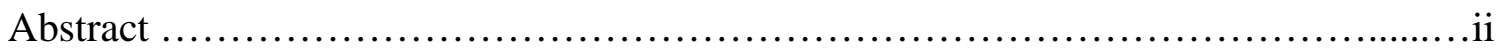

Acknowledgements ................................................................

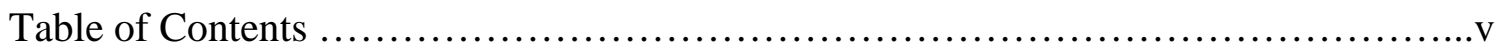

List of Acronyms....................................................................

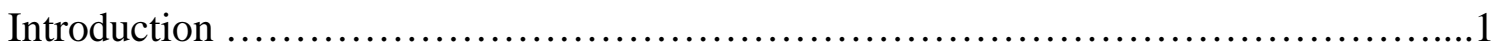

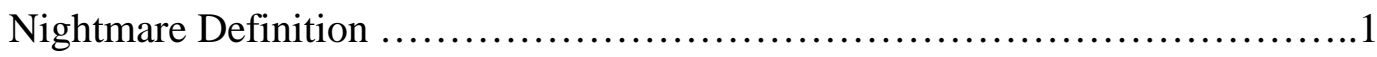

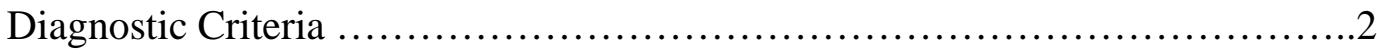

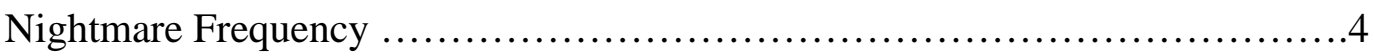

Nightmare Theories ...........................................................

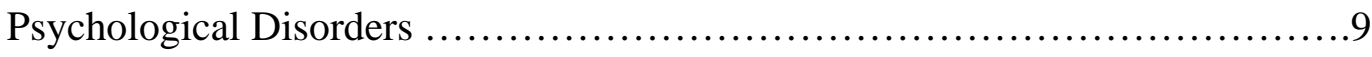

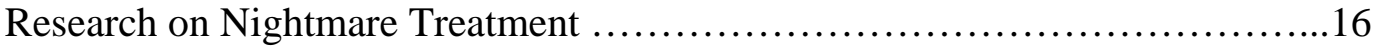

Statement of the Problem .................................................28

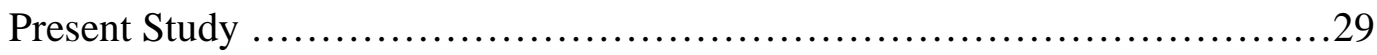

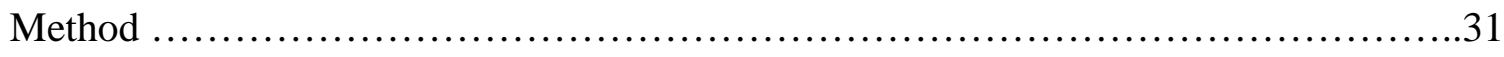

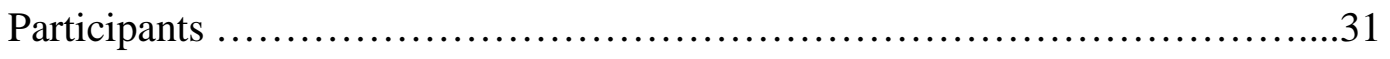

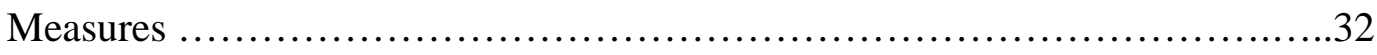

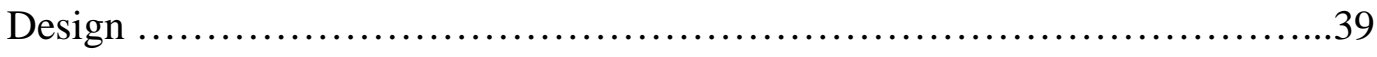

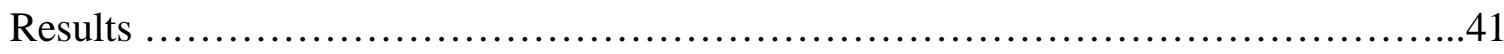

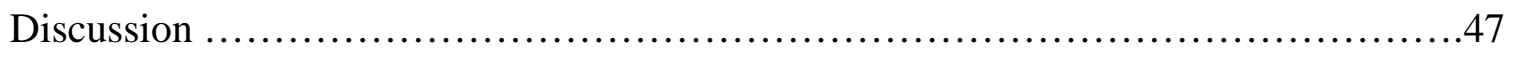

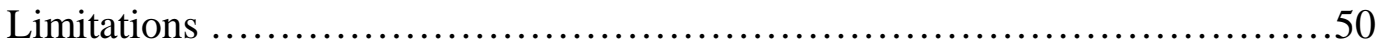

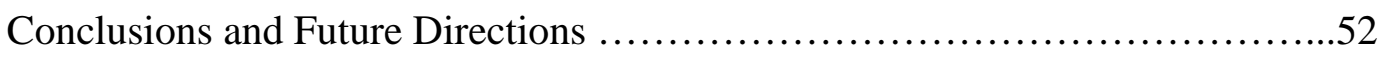

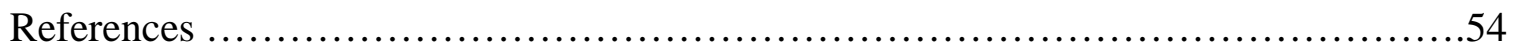




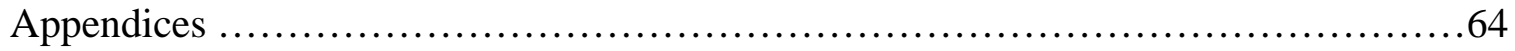

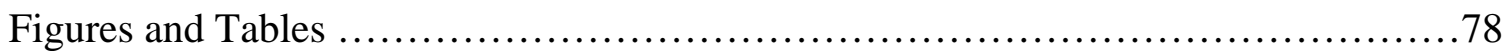




\section{List of Acronyms}

IRT: Imagery Rehearsal Therapy

PTSD: Post-Traumatic Stress Disorder

REM: Rapid Eye Movement

DSM: Diagnostic and Statistical Manual

ICD: International Classification of Diseases

ICSD: International Classification of Sleep Disorders

AMPHAC/AND: amygdala, medial prefrontal cortex, hippocampus, anterior cingulate cortex, affect network dysfunction

PRT: Personal Rapid Transit

IRB: Institutional Review Board

APA: American Psychological Association

BAI: Beck Anxiety Inventory

BDI: Beck Depression Inventory

BHS: Beck Hopelessness Scale

BSS: Beck Scale for Suicide Ideation

DDNSI: Disturbing Dreams and Nightmares Severity Index

ISI: Insomnia Severity Index

MAST/AD: Michigan Assessment-Screening Test for Alcohol and Drugs

PANAS: Positive and Negative Affect Scales

PCL: PTSD Checklist - Civilian Version

CAPS: Clinician-Administered PTSD Scale 
Nightmares are linked to several negative outcomes, including suicide attempts and death by suicide (Sjöström, Hetta, \& Wærn, 2009; Tanskanen et al., 2001). Further, nightmares are associated with psychological disorders such as post-traumatic stress disorder (Harvey, Jones, \& Schmidt, 2003), anxiety symptoms (Levin \& Nielsen, 2007), depressive symptoms (Besiroglu, Agargun, \& Inci, 2005), schizophrenia (Hartmann1981), dissociative disorders (Agargun, Kara, Ozer, Selvi, Kiran, \& Ozer, 2003), and borderline personality disorder (Hartmann, 1981). Research has provided evidence that nightmares even increase the risk of developing PTSD (Mellman, David, Kulick-Bell, \& Hebding, 1995), and engaging in future suicide attempts in a sample of suicide attempters (Sjöström, et al., 2009). Despite these associations, research examining nightmare treatments is still in its infancy. The current study investigated Imagery Rehearsal Therapy (IRT), a particularly promising nightmare treatment. The current paper will first present the definition of nightmares and diagnostic criteria for nightmare disorder. Then, the paper will examine the prevalence of nightmares, as well as their associations with psychopathology. Lastly, there will be a review of the research on nightmare treatments, followed by the current investigation of IRT for nightmares.

\section{Nightmare Definition}

Nightmares, defined as vivid, disturbing, or frightening dreams that awaken the individual, are a common form of parasomnia (Levin \& Nielsen, 2007). Nightmares primarily occur during REM sleep, and hence are more common during the second half of the night (American Academy of Sleep Medicine, 2006). The fact that nightmares occur in REM sleep differentiates them from night terrors, a parasomnia that occurs in non-REM sleep. Following a nightmare, an individual is usually fully alert and may have difficulty returning to sleep. Although there are several sets of criteria for nightmare disorder (discussed later), most require 
an individual to have recurrent nightmares that awaken the individual to a state of alertness (American Academy of Sleep Medicine, 2001, 2006; American Psychiatric Association, 2000).

Disturbing dreams, which are negative dreams that do not lead to a startled awakening, are usually excluded from the definition of nightmares due to their lack of a startled awakening. However, disturbing dreams and nightmares are quite similar, as both require awakening from a negative dream. Although an awakening is not required by the definition of disturbing dreams, it is implicit as one must awaken to remember a dream. Thus, the only difference between nightmares and disturbing dreams is whether or not the awakening from the dream was startled. Due to their similarities, the majority of nightmare studies, including the current study, combine disturbing dreams and nightmares (Levin \& Nielsen, 2007).

\section{Diagnostic Criteria}

Currently, there is no single agreed upon set of criteria for diagnosing nightmare disorder. Therefore, three of the most-used criteria for nightmare disorder are presented here. Although they have significant differences, all three require (or imply) that a nightmare must suddenly awaken the individual.

The Diagnostic and Statistical Manual of Mental Disorders, $4^{\text {th }}$ edition text revision (American Psychiatric Association, 2000) requires the following for the diagnosis of a nightmare disorder:

A. Repeated awakenings from the major sleep period or naps with detailed recall of extended and extremely frightening dreams, usually involving threats to survival, security, or self-esteem. The awakenings generally occur during the second half of the sleep period. 
B. On awakening from the frightening dreams, the person rapidly becomes oriented and alert (in contrast to the confusion and disorientation seen in Sleep Terror Disorder and some forms of epilepsy).

C. The dream experience, or the sleep disturbance resulting from the awakening, causes clinically significant distress or impairment in social, occupational, or other important areas of functioning D. The nightmares do not occur exclusively during the course of another mental disorder (e.g., delirium, Post-traumatic Stress Disorder) and are not due to the direct physiological effects of a substance (e.g. a drug of abuse or medication) or a general medical condition (pg. 634)

The DSM criteria are quite restrictive, as an individual must not qualify for posttraumatic stress disorder or sleep terror disorder in order to meet criteria for a nightmare disorder diagnosis. Therefore, a large number of individuals with clinically-significant nightmares will not meet DSM criteria for nightmare disorder.

The International Classification of Diseases (ICD-10) has the simplest criteria of the three. It defines nightmare disorder as follows:

Dream experiences loaded with anxiety or fear. There is very detailed recall of the dream content. The dream experience is very vivid and usually includes themes involving threats to survival, security, or self-esteem. Quite often there is a recurrence of the same or similar frightening nightmare themes. During a typical episode there is a degree of autonomic discharge but no appreciable vocalization or body motility. Upon awakening the individual rapidly becomes alert and oriented (World Health Organization, 2007). 
Unlike the DSM, the ICD-10 does not exclude individuals with a diagnosis of posttraumatic stress disorder or night terror disorder from receiving a nightmare disorder diagnosis.

The International Classification of Sleep Disorders - Second Edition (ICSD-2; American Academy of Sleep Medicine, 2006) criteria differ from the DSM criteria in that an individual may have post-traumatic stress disorder or night terrors and still meet criteria for nightmare disorder. The ICSD’s criteria for nightmare disorder are as follows:

A. Recurrent Episodes of awakenings from sleep with recall of intensely disturbing dream mentation, usually involving fear or anxiety, but also anger, sadness, disgust, or other dysphoric emotions.

B. Full alertness on awakening, with little confusion or disorientation; recall of sleep mentation is immediate and clear

C. At least one of the following associated features is present:

1. Delayed return to sleep after the episode

2. Occurrence of episodes in the latter half of the habitual sleep period (p. 156)

Like the ICD-10, the ICSD-2 does not preclude individuals with post-traumatic stress disorder from receiving a diagnosis of nightmare disorder.

\section{Nightmare Frequency}

Nightmares are often thought of as a disorder of childhood (Levin \& Nielsen, 2007). It is estimated that $10-50 \%$ of children age three to six experience occasional nightmares (American Psychiatric Association, 2000), with approximately 19\% of children experiencing nightmares at least once per week (Schredl, Biemelt, Roos, Dunkel, \& Harris, 2008). Although nightmares 
are often viewed as a childhood sleep disorder, research suggests that nightmares may persist into adulthood, and are a function of age and sex. In a sample of college students, 53\% of participants reported having nightmares at least once a month and 14\% reported nightmares at least once per week (Nadorff, Nazem, \& Fiske, 2011).

In a large study of nightmare frequency, Nielsen, Stenstrom, and Levin (2006) collected nightmare information from 23,990 individuals using an online questionnaire and found that women reported significantly more nightmares than men (4.44 per month vs. 3.39). For women, nightmare frequency increased from ages 10 - 19 to 20 - 39 and then decreased. However, for men, nightmare frequency remained stable from ages 10-19 through 30-39, and then decreased after that. Thus, research suggests that the prevalence of nightmares is partially dependent upon age and sex (Nielsen, et al., 2006).

Studies examining nightmares in adults have found nightmare disorder prevalence rates ranging from $2 \%-6 \%$. This prevalence range is highly consistent across cultures, with similar rates having been found in the United States, Canada, France, Iceland, Sweden, Belgium, Finland, Austria, Japan, and the Middle East (Levin \& Nielsen, 2007). Very little research has examined the prevalence of nightmares among older adults. However, the research that has been done suggests that nightmares may be less common among older adults compared with children and younger adults. In a study comparing older adults and college students, only $4.3 \%$ of older adults reported having a problem with nightmares, which was significantly less than the $19.5 \%$ of college students in the study reporting having a nightmare problem (Salvio, Wood, Schwartz, \& Eichling, 1992).

Nightmare Theories 
It is often helpful to have a theoretical framework in order to understand a disorder and its effects. However, nightmares have historically received little attention from theorists or researchers. Even Freud, who did a considerable amount of work on dreams, rarely discussed nightmares. In his book The Interpretation of Dreams, Freud only mentions nightmares twice, and neither time did he provide a theory as to why individuals have nightmares (Freud, 2004). Contemporary theorists have put forth ideas about the etiology of nightmares, although little evidence supporting the theories exists. Despite this fact, a review of the current theories is still warranted, especially given that some of the nightmare treatments are based upon these theories.

After studying the histories of many nightmare sufferers from the psychoanalytic perspective, Hartmann (2001) concluded that nightmares are caused by "thin boundaries.” Thin boundaries refer to "the lack of separation between areas and processes in the mind, and also a lack of walls and defense” (Hartmann, 2001). Hartmann (2001) came to this conclusion based on the notion that trauma more strongly affects individuals with thin boundaries, and since nightmares are a manifestation of trauma, thin boundaries must be associated with nightmares. Supporting this theory, Hartmann (1989) found that thin boundaries (as defined by the Hartmann Boundaries Questionnaire) were positively correlated with remembering one’s dreams, which may lead to remembering one’s nightmares. Similarly, other researchers have also found that thin boundaries are associated with dream recall, more negative and emotionally intense dreams, and regarding one's dreams to be meaningful (Schredl, Schafer, Hofmann, \& Jacob, 1999).

Cartwright (2001) articulated a different view of nightmares, focusing on emotion processing instead of boundaries. She stated that when a traumatic event occurs, an individual may be unable to handle all of the resulting emotions at that time. Therefore, nightmares may emerge in order to help process the emotions caused by the trauma. For most individuals, the 
nightmares will subside once the processing has completed, but for others, the nightmares can become a chronic problem. Although other theorists agree that emotion regulation may help explain nightmares (Levin \& Nielsen, 2009), to date no research has been published linking emotion regulation problems with nightmares.

Taking a more biological approach, Levin and Nielsen (2007) published the AMPHAC/AND Neurocognitive model of disturbed dreaming. The model seeks to explain disturbed dreaming at both physiological and cognitive levels. The neurophysiological component of the model, called the AMPHAC network, specifies the involvement of the following brain structures: "the amygdala (A), the medial prefrontal cortex (MP), the hippocampus (H), and the anterior cingulate cortex (AC)" (Levin \& Nielsen, 2007, p. 84). The cognitive component of the model is called affect network dysfunction (AND) and is comprised of affect load (AL) and affect distress (AD). Affect load is defined as the amount of stressful or negative emotional events a person is experiencing, and affect distress is an individual's predisposition to react strongly to emotional stimuli. The model posits that the physiological and cognitive levels serve the function of fear-memory extinction during normal dreaming. In this process, components of fearful events are combined in a new way with non-fearful memories, disarming the memories. However, for individuals with significant distress (especially those who are predisposed to be sensitive to distress), the fearful memories may not be altered, or may be altered in a way even more frightening than the original memory, leading to disturbing dreams (Levin \& Nielsen, 2009). To date, there is no evidence directly supporting the AMPHAC/AND Neurocognitive model of disturbed dreaming. However, the lack of evidence may be due to the recent publication of the model. Furthermore, the AMPHAC/AND model is most consistent with the activation-synthesis hypothesis of dreaming (Hobson \& McCarley, 1977) which is one 
of the most widely-accepted theories of normal dreaming. The activation synthesis hypothesis postulates that dreaming is an event that is physiologically shaped by the sections of the brain that are activated during sleep. In an attempt to make sense of the activation, the brain synthesizes the impulses into a narrative, which we experience as dreams.

Nightmares have also been viewed through a cognitive-behavioral lens. The cognitivebehavioral theory of nightmares posits that nightmares cause sleep avoidance behaviors (Krakow, Hollifield, et al., 2001; St-Onge, Mercier, \& De Koninck, 2009). In this theory, the nightmare is considered to be a conditioned stimulus that causes a conditioned avoidance response. The awakening then reinforces the belief that the only way to avoid nightmares is to remain awake, which leads to the nightmare sufferer developing other behaviors to avoid sleep. However, there are several limitations to the cognitive-behavioral theory of nightmares. First, although it explains why nightmare sufferers may sleep less and have poor sleep quality, it does not provide an explanation for why people have nightmares. Second, the theory is not fully consistent with behavioral theory.

It stands to reason that individuals become aware of their nightmares due to awakenings, which are a learned behavior that has been negatively reinforced by waking up during nightmares, and thereby avoiding the negative content. Put simply, an individual may wake up and thereby escape from having the nightmare, which negatively reinforces the awakenings that are serving as an avoidance response. However, the avoidance of the nightmare increases sleep fragmentation, making an individual more likely to remember their dreams, perpetuating the nightmare problem. Additionally, it is possible that avoiding the nightmare may prevent exposure to the nightmare, potentially explaining why nightmares persist. 
To date, all of the non-pharmacological treatments for nightmares are based on the cognitive-behavioral theory of nightmares. Studies have shown significant reductions in nightmares through use of systematic desensitization (Cellucci \& Lawrence, 1978), exposure (Burgess, Gill, \& Marks, 1998), and imagery rehearsal therapy (Krakow, Hollifield et al. 2001), as discussed below.

\section{Psychological Disorders}

In addition to understanding the theories of nightmares, it is important to also know the relation between nightmares and related disorders, as these relations may affect the development and treatment of nightmares. Additionally, nightmares may be a precursor to psychopathology (Mellman, et al., 1995; Ohayon \& Shapiro, 2000), and as such nightmares have great clinical relevance.

Although the most well-known comorbidity is post-traumatic stress disorder, nightmares are also related to several other psychological disorders. The next section will review the literature examining disorders that are often comorbid with nightmares.

\section{Post-traumatic stress disorder}

Nightmares have a strong association with post-traumatic stress disorder (Harvey, et al., 2003). The nightmares observed in PTSD (sometimes referred to as traumatic nightmares) are often re-enactments of the traumatic event, and are consistent with both the re-experiencing and hyperarousal criteria of PTSD (American Psychiatric Association, 2000). In addition to being common in PTSD, nightmares are a chronic symptom of PTSD. Two studies examining war veterans found that nightmares persisted up to 50 years following the traumatic experience (Guerrero \& Crocq, 1994; Kaup, Ruskin, \& Nyman, 1994). 
Nightmares are not only related to PTSD, but they may influence the development of PTSD. PTSD symptoms are more severe among individuals who reported having nightmares prior to the traumatic event than among individuals who did not report experience nightmares prior to the trauma (Mellman, et al., 1995). Similarly, Ohayon and Shapiro (2000) found that insomnia (60.9\%) and nightmares (40\%) preceded trauma, and the subsequent development of PTSD, among a large proportion of the sample. These finding suggest that nightmares and insomnia may be risk-factors for developing PTSD later in life. They also suggest that there may be a certain vulnerability that both sleep difficulties and PTSD share.

For individuals who have been diagnosed with PTSD, nightmares have been shown to be associated with poorer outcomes. Mellman and colleagues (2001) followed 60 individuals who experienced a life-threatening trauma. They found having nightmares of the trauma shortly after the trauma were related to more severe PTSD symptoms six weeks later. Conversely, individuals with PTSD rate their nightmares as being more distressing than nightmare sufferers without PTSD (Germain \& Nielsen, 2003).

In sum, nightmares and PTSD are closely related. The fact that nightmare and PTSD onset and severity have a reciprocal relation leads one to wonder if there is a common vulnerability that underlies both disorders. As such, it is possible that treating one disorder may help the other.

\section{Anxiety Symptoms}

Nightmares are also related to anxiety symptoms in children, adolescents, adults, and psychiatric inpatients (Levin \& Nielsen, 2007). In particular, nightmares are related to death anxiety, fear of annihilation, and neuroticism (Levin \& Nielsen, 2007). Although few studies have separated nightmare frequency from severity, the studies that have suggest that the relation 
between nightmares and anxiety symptoms may be driven by nightmare severity, not frequency. Levin and Fireman (2002) found that nightmare severity correlated much more strongly with symptoms of depression and anxiety than with nightmare frequency.

Although research has demonstrated an association between nightmares and anxiety symptoms, much is not known. To date, research has not examined if nightmares precede or follow anxiety symptoms. Knowing the precedence of disorders may help in understanding the etiology of both nightmares and anxiety disorders. This is important for nightmare treatment as the efficacy of nightmare treatments may differ depending upon whether nightmares are the primary problem, or result from the anxiety. For example, if nightmares are due to high levels of anxiety, and the anxiety remains untreated, nightmares may not be reduced by nightmare treatments due to the core problem being anxiety.

\section{Depressive Symptoms}

Although sparse, research has demonstrated a link between nightmares and depressive symptoms. The majority of the research investigating nightmares and depression has focused on the relation between nightmares and melancholic features within depression. Besiroglu, Agargun, and Inci (2005) found that nightmares were significantly more common among depressed individuals with melancholic features than among those without melancholic features. Further, depressed suicide attempters with melancholic features have been shown to have more nightmares than suicide attempters without melancholic features (Agargun et al., 2007).

Very little research has examined whether having nightmares increases the likelihood of having depression. Utilizing a multiple regression analysis, Levin and Fireman (2002) found that nightmare distress was significantly related to higher levels of depressive symptoms. Nightmare frequency was not significantly related to depressive symptoms after statistically 
controlling for nightmare distress. Similar to anxiety, further research is needed to explore the precedence of nightmares and depression, as the precedence may influence treatment outcomes.

\section{Schizophrenia}

In line with Hartmann’s thin boundaries theory, research has demonstrated a link between nightmares and schizophrenia-spectrum disorders. Hartmann (1981) examined 38 adults who reported having at least one nightmare per week. Four met DSM-III criteria for schizophrenia (11\%) and six met criteria for schizotypal personality disorder (16\%). These rates are higher than the rate of schizophrenia $(0.5 \%-1.5 \%)$ and schizotypal personality disorder $(3 \%)$ found in the general population (American Psychiatric Association, 2000). However, it should be noted that while not an inpatient sample, nearly 75\% of Hartmann’s sample had received psychiatric services in the past, and 10\% had a prior inpatient hospital stay. Further, Herz and Melville (1980) found that nightmares are a common precursor of relapse of schizophrenia.

Nightmare sufferers also have elevated scores on measures validated to detect schizophrenia. Hartmann and colleagues (1987) found that nightmare sufferers scored significantly higher on the Minnesota Multiphasic Personality Inventory subscales associated with psychosis. Similarly, Levin (1998) demonstrated that nightmare sufferers had greater levels of schizotypy than individuals in the control group.

Although the mechanism is not clear, a strong link exists between nightmares and schizophrenia. Although untested, the relation between nightmares and schizophrenia may help explain why some researchers have found that individuals with PTSD are more likely to have comorbid schizophrenia (Davidson, Hughes, Blazer, \& George, 1991).

\section{Dissociative Disorders}


Dissociative disorders have been linked to acute stress disorder and post-traumatic stress disorder (Agargun, Kara, Ozer, Selvi, Kiran, \& Kiran, 2003), suggesting they may be associated with nightmares as well. A few studies support a link between dissociative disorders and nightmares. Agarun and colleagues (2003) found that 57\% of individuals with a dissociative disorder met criteria for a nightmare disorder. This rate is much higher than the $2-7 \%$ prevalence of nightmare disorder found in community samples (Levin \& Nielsen, 2007). Individuals who report nightmares also have more dissociative symptoms than do individuals without nightmares. In a sample of 292 undergraduate students (age 16 - 21), those who reported having nightmares scored significantly higher on the Dissociative Experiences Scale than those who did not report having nightmares (Agargun, Kara, Ozer, Selvi, Kiran, \& Kiran, 2003). Similarly, Semiz, Basoglu, Ebrinc, and Cetin (2008) found a significant correlation (0.43) between the Dissociative Experiences Scale and nightmare frequency among individuals with borderline personality disorder.

To date, it is not clear why nightmare sufferers are more likely to experience dissociative symptoms, nor is it clear if this relation holds for nightmare sufferers who have not experienced a traumatic event. Theoretically, it makes sense that dissociative disorders and nightmares are related because both are believed to be associated with having thin boundaries (Hartmann, 1989). If this is a common thread, an intervention that strengthens boundaries may be beneficial for both nightmares and dissociative symptoms in those who suffer from both.

\section{Borderline Personality Disorder}

Researchers have also found a link between nightmares and borderline personality disorder. In Hartmann's (1981) sample of 38 adults with weekly nightmares, he found that nine of the participants (24\%) met DSM-III criteria for borderline personality disorder. This rate is 
higher than the rate of borderline personality disorder (2\%) found in the general population (American Psychiatric Association, 2000). Similarly, Claridge, Davis, Bellhouse, and Kaptein (1998) found that nightmare distress correlated strongly (0.42) with the borderline personality scale in a sample of 60 undergraduate women.

Individuals with borderline personality disorder have also been shown to report more nightmares than those without borderline personality disorder. Semiz, Basoglu, Ebrinc, and Cetin (2008) compared a sample of 88 participants diagnosed with borderline personality disorder with age and sex-matched controls. The patients with borderline personality disorder reported more nightmares, greater dream anxiety, and more disturbed sleep than the control participants. Further, those with borderline personality disorder and a nightmare disorder exhibited more severe psychopathology than those with borderline personality disorder but without a nightmare disorder.

The relation between nightmares and borderline personality disorder is interesting, as it may help explain the findings that nightmares are associated with suicidal behavior (Cukrowicz et al., 2006; Nadorff, et al., 2011; Sjöström, et al., 2009; Sjöström, Wærn, \& Hetta, 2007; Tanskanen, et al., 2001). Borderline personality disorder has been associated with several negative outcomes, including suicidal behavior. Suicidal behavior is common in the disorder, with approximately $10 \%$ of those diagnosed with borderline personality disorder dying by suicide (Paris, 2002), and suicidal behavior is one of the DSM-IV criteria for the disorder (American Psychiatric Association, 2000). To date, no study has examined whether or not the relation between nightmares and suicide is mediated by borderline personality disorder.

\section{Suicide}


A substantial and growing literature suggests that nightmares increase one’s risk of suicidal behavior. Cukrowicz and colleagues (2006) found that disturbing dreams or nightmares were significantly related to suicidal ideation in a college sample (Mean age = 19.2 years). Multiple regression revealed that nightmares were associated with suicidal ideation independent of insomnia symptoms. Similarly, we (Nadorff, et al., 2011) examined the relation between nightmares and suicidal ideation in a sample of 583 college students (Mean age $=19.4$ years). We found that nightmares were associated with suicidal ideation after statistically controlling for the symptoms of depression, anxiety, and post-traumatic stress disorder, $B=0.021, S E=.008, p$ $=.01$.

Nightmares have also been examined in relation to suicide attempts. Sjostrom, Waern, and Hetta (2007) studied 165 patients age 18 to 68 years $(M=35.3$ years) who had been admitted to the hospital following a medically serious suicide attempt. Although insomnia was the most common sleep complaint, two-thirds of participants also reported having nightmares. A regression analysis revealed that onset insomnia, terminal insomnia, and nightmares were all significantly associated with higher scores of suicidality, defined as the risk of attempting suicide. Nightmares remained significantly related to high suicidality after controlling for DSM axis I disorders, posttraumatic stress disorder diagnoses, depression symptoms, and anxiety symptoms, adjusted $O R=3.50,95 \% C I=1.57-7.81, p<.01$. Further, a follow-up study found that persistent nightmares predicted suicide attempts in the next two years in the same sample after controlling for DSM Axis I disorders, adjusted $O R=4.18,95 \% C I=1.47-11.89, p<.01$ (Sjöström, et al., 2009).

Nightmares are also related to death by suicide. Tanskanen et al. (2001) examined the relation between nightmares and death by suicide in a prospective study conducted in Finland. 
When compared with individuals without nightmares, those reporting occasional nightmares were at $57 \%$ greater risk of death by suicide. Further, participants reporting frequent nightmares were at $107 \%$ greater risk of suicide compared to those without nightmares. This study suggests that nightmares are potentially a risk factor for suicide.

Although nightmares are associated with several disorders, temporal precedence has not been investigated. Therefore, it is unknown whether nightmares cause other disorders, or whether the nightmares are a product of the other disorders. This is important, because the presence of co-morbid disorders may affect the efficacy of nightmare treatment. Further, few studies have examined nightmare disorder diagnosis, instead utilizing nightmare frequency or severity ratings.

\section{Research on Nightmare Treatment}

Four main types of psychosocial interventions have been used in the literature to treat nightmares: exposure, systematic desensitization, lucid dreaming, and imagery rehearsal therapy. This section will summarize the research that has been conducted on each of these four interventions to examine their efficacy as well as their strengths and limitations.

\section{Systematic Desensitization}

Systematic desensitization, a gradual exposure method where an individual utilizes relaxation techniques while being exposed to a hierarchy of fears, was one of the first treatments used to treat nightmares (Cellucci \& Lawrence, 1978; Miller \& DiPilato, 1983). Cellucci and Lawrence (1978) recruited a sample of undergraduate students who retrospectively reported having at least two nightmares per week. These undergraduates were asked to track their dreams, and those who reported having at least one nightmare per week were enrolled in the study $(\mathrm{N}=29)$. The participants were asked to log when they had a nightmare and the distress it 
caused (10-point scale) over the course of 8 weeks. Then the participants were randomly assigned to either five sessions of systematic desensitization, placebo (a nightmare discussion group), or continuous tracking of nightmares. The treatment consisted of five sessions. Systematic desensitization and the placebo group both resulted in a significant reduction in nightmare frequency. However, the systematic desensitization group improved significantly more than the placebo group in nightmare frequency and severity.

Miller and DiPilato (1983) investigated using systematic desensitization to treat 32 selfreferred nightmare sufferers. The sample was mostly female and averaged having nine nightmares per month. The authors stated that individuals with major psychopathology were excluded, but did not state what constituted major psychopathology. The participants were randomly assigned to six weekly sessions of relaxation training, systematic desensitization, or a waitlist control. Immediately following treatment, there was a significant reduction in nightmare frequency, but not intensity, for both systematic desensitization and relaxation training when compared with the waitlist control group. However, 25 weeks post-treatment, nightmare intensity was also found to be significantly reduced in the systematic desensitization group.

These two studies support of the use of systematic desensitization for nightmares. However, there are a few limitations: systematic desensitization requires several additional sessions (5-6 sessions vs. 1 for IRT), and it may be perceived as more aversive than IRT or lucid dreaming to the client due to the client being exposed to more of the negative content of the nightmare when compared to IRT or lucid dreaming. Nonetheless, systematic desensitization appears to be promising, despite these limitations.

\section{Exposure}


Treating nightmares through exposure involves confronting an individual with his or her nightmare until it is no longer causing the individual significant distress. To date, only two studies have evaluated the efficacy of treating nightmares using exposure therapy (Burgess, et al., 1998; Grandi, Fabbri, Panattoni, Gonnella, \& Marks, 2006). However, both of these studies have found that exposure treatment results in significant reductions in nightmares.

Burgess and colleagues (1998) investigated the efficacy of using a self-administered exposure treatment. They recruited 107 nightmare sufferers through media advertisements. Potential participants were then mailed questionnaires to assess if they met DSM-III-R criteria for recurrent nightmares. In order to be included in the study, participants had to be at least 18 years of age, have nightmares for at least one year, have the nightmares occur at least weekly over the last 6 months, and have scores greater than 5 out of 11 on distress and sleep disturbance caused by the nightmares. The exclusion criteria were severe psychiatric illness, night terrors, narcolepsy, sleep paralysis, asthma, taking a medication that is likely to cause nightmares, and drinking more than two (three for men) alcoholic drinks per day. The participants meeting these criteria were randomized into either a self-exposure, self-relaxation, or wait-list control group. Similar to the previous study, participants spent between 30 and 60 minutes daily for a period of four weeks doing the self-exposure treatment. Participants in the self-exposure group had a significantly greater reduction in nightmares $(d=1.18)$ at one and six months post-treatment when compared with the self-relaxation and wait-list groups. Additionally, those who received the self-exposure treatment also experienced a significant reduction in their depressive symptoms $(d=.76)$.

Grandi and colleagues (2006) also investigated the efficacy of a self-exposure treatment for nightmares. They recruited a sample of 10 nightmare sufferers who met DSM criteria for 
nightmare disorder. Since the DSM criteria were used, all DSM axis I and II disorders other than nightmares and medications that potentially cause nightmares were exclusion criteria. The authors had the participants track their nightmares over three months and then taught the participants how to do self-directed exposure in the morning following having a nightmare. The participants were asked to work for 30-60 minutes in a workbook for a period of four weeks and then participated in follow ups immediately post-treatment as well as 3, 4, 5, 7, 10, 16, 28, and 52 months post-treatment. The authors found that the exposure treatment significantly reduced not only nightmare frequency and intensity, but also symptoms of anxiety, depression, and sleeplessness at the post-treatment follow-up. Remarkably, most of the gains were maintained over four years.

Both studies that examined use of self-administered exposure found strong positive results. However, both relied on participants doing a significant amount of homework each day (30-60 minutes), which may affect compliance and may even be impossible for some nightmare sufferers. Also, similar to systematic desensitization, exposure requires the client to reexperience the entire nightmare, instead of only part of the nightmare as in IRT, and hence may be more aversive than IRT or lucid dreaming. Overall, self-exposure therapy appears to be a promising treatment, although it is limited due to requiring a significant amount of homework and the possibility that it may be aversive.

\section{Lucid Dreaming Therapy}

The lucid dreaming approach to nightmares differs significantly from exposure and systematic desensitization. Instead of exposing an individual to his/her nightmare in order to reduce nightmare severity, lucid dreaming therapy involves helping individuals realize when they are dreaming and, once this realization is made, helping them learn how to change their 
dream while they are having it. In lucid dreaming therapy, participants are trained to be able to identify and change their dream while they are dreaming. Some strategies used to train lucid dreaming are testing reality and identifying “dreamsigns” which are events that do not occur in real life (The Lucidity Institute, 1993). Once able to identify when they are dreaming, the participants are instructed to actively change their dream, while experiencing it, so the ending is positive. Thus, positive ending is not designed beforehand, nor is it rehearsed, as is done in IRT, but rather the participant determines the ending while asleep (Spoormaker \& van den Bout, 2006).

Lucid dream frequency has been shown to be related to nightmare frequency, and it has been hypothesized that nightmares may trigger lucid dreaming (Schredl \& Erlacher, 2004). Given that individuals with nightmares may be more likely to have lucid dreams (Schredl \& Erlacher, 2004), and those who do not already have lucid dreams can be taught (Purcell, Mullington, Moffitt, \& Hoffmann, 1986), it makes sense to try to train nightmare sufferers to become lucid and change their nightmare while the nightmare is occurring.

To date, there have only been two studies investigating the efficacy of lucid dreaming therapy on nightmares (Spoormaker \& van den Bout, 2006). In the first study, 23 nightmare sufferers were randomly assigned to either a two-hour individual lucid dreaming treatment session, a two-hour group lucid dreaming treatment session, or a waitlist control group. Homework was utilized following treatment, although the type, frequency, and duration of the homework were not mentioned. At post-test (12 weeks after treatment), participants receiving both the individual and group treatment reported significantly fewer nightmares when compared to pre-treatment baseline. However, there was no significant difference between the treatment and control groups, and no significant change in sleep quality or PTSD symptom severity. In the 
second study, Lancee and colleagues (2010) compared IRT, IRT and sleep hygiene, IRT and lucid dreaming, and waitlist control. Surprisingly, IRT alone was shown to be more effective than the other two treatment conditions, and was the only treatment condition that resulted in a significant improvement in nightmares compared to the control group. However, the authors state that the lack of significant results for the other conditions may have been due to low power and high rates of attrition.

The lucid dreaming approach differs significantly from the other nightmare treatments. Instead of trying to reduce the frequency or severity of nightmares, it attempts to stop nightmares mid-way through. A strength of lucid dreaming is it more pleasant to the client than exposure or systematic desensitization, although some aspects of exposure are present, as one has to imagine their dream as part of the treatment. Nonetheless, the research findings thus far are weak, with individuals receiving the lucid dreaming treatment failing to out-perform the wait-list control group. Thus, based on the current literature, lucid dreaming is not a good option for treating nightmares.

\section{Imagery Rehearsal Therapy}

Imagery rehearsal therapy (IRT) is by far the most widely researched of the nightmare treatments with eleven articles having been published on its efficacy. It may also be one of the most pleasant treatments, as the client is not repeatedly exposed to their full nightmare. In imagery rehearsal therapy, the client is instructed to imagine their nightmare but change the ending in any way they want. Once they have done this, they are encouraged to rehearse the new dream at least once a day for at least a week. Over time, it is expected that the nightmare will change from the old nightmare into a new dream. 
Imagery rehearsal therapy is based on the theory that nightmares are "a learned behavior" (Krakow, 2002, pg 18) and, as such, can be replaced by a more pleasant behavior (in this case a new dream) that achieves the same function. The client practices a new dream through imagery exercises, and over time, a new dream replaces the old nightmare. Another possible explanation for IRT's effectiveness can be found in the activation-synthesis model (Hobson \& McCarley, 1977), which posits that dreams are the byproduct of events based on waking experiences. By imagining the new dream daily, the new dream content may be incorporated into one’s dreams due to it being part of one’s daily experience.

The first investigation of imagery rehearsal therapy consisted of 20 individuals who were randomly assigned to treatment or to nightmare recording (Neidhardt, Krakow, Kellner, \& Pathak, 1992). Imagery rehearsal therapy was administered in one session, with participants being instructed to practice the imagery once a day for at least three days for each nightmare that they desired to change. The authors followed up with all participants three months after treatment. The authors found that nightmare frequency significantly decreased in both groups, but there was no significant difference between the imagery rehearsal therapy and recording groups ( $d=1.28$ for IRT, 0.67 for recording). However, given that the effect size for the recording group was fairly large, one has to wonder whether the study was adequately powered. Symptoms of depression $(d=0.82)$ and anxiety $(d=0.62)$ were both significantly reduced in the imagery rehearsal therapy group. After 30 weeks, both the IRT and recording group (which received IRT three months after the IRT group) demonstrated significant decreases in nightmare frequency (the authors did not state if these groups significantly differed). However, only the imagery rehearsal therapy group had a significant decrease in nightmare severity after 30 weeks (Krakow, Kellner, Neidhardt, Pathak, \& Lambert, 1993). 
Building upon the promising results of the first study, Krakow and colleagues (1996) examined IRT utilizing a sample of 58 chronic nightmare sufferers who had nightmares at least once per week. In this uncontrolled trial participants received a single, 2.5 hour group IRT session. The participants were instructed to practice the imagery of their new dream at least once a day following treatment. The researchers then followed up with the participants after three and 18 months. The authors found a significant decrease in nightmare frequency from baseline to three and 18 month follow-up. They also found that there was a significant decrease in nightmare frequency from three months following treatment to 18 months suggesting that the patients continued to improve more than three months after treatment. In addition to decreases in nightmare frequency, anxiety symptoms were significantly reduced $(d=0.4)$. However, depressive symptoms were not significantly reduced between pre-treatment and 18-month follow-up $(d=0.18)$.

In the first study to investigate imagery rehearsal therapy in a sample with PTSD, Krakow and colleagues (2000) conducted a large randomized controlled trial utilizing 169 female survivors of sexual assault with PTSD. The 87 participants receiving the treatment received three group sessions of imagery rehearsal therapy over a period of five weeks. After three months, nightmares frequency was significantly reduced in the treatment group when compared with the wait-list control group $(d=1.2)$. In addition to nightmare frequency being reduced, PTSD symptoms were also significantly reduced $(d=1.2)$ in the group that received treatment. This finding suggests that imagery rehearsal therapy may be an effective treatment for PTSD symptoms in those with PTSD and nightmares.

Krakow and colleagues (2001a) attempted to replicate the previous study with another large randomized controlled trial of imagery rehearsal therapy in women with PTSD. In this 
study of 168 female participants with PTSD, 88 were randomized into the treatment condition and 80 into the waitlist control. In addition to nightmare frequency, the authors assessed sleep quality and PTSD symptoms. The participants received three sessions of imagery rehearsal therapy and were instructed to practice the imagery of their new dream for between 5 and 20 minutes per day. Further, participants were instructed to not try to change more than two dreams per week. The researchers conducted a follow-up with participants after three and six months. The authors found that the treatment group had significantly reduced nightmares $(d=0.85)$, improved sleep quality $(d=0.67)$, and reduced PTSD symptoms $(d=1.0)$ relative to the control group.

Krakow and colleagues (2001b) further extended the IRT findings by testing it in a sample of adolescent girls who had suffered a sexual assault. The sample consisted of 19 adolescent girls at a treatment facility who were randomly assigned to either the treatment $(n=9)$ or control $(n=10)$ group. The treatment group received IRT in a six hour workshop conducted over one day. The treatment group was instructed to practice the imagery for 5 to 20 minutes every day. The control group received no intervention. After three months, the treatment group reported a $71 \%$ decrease in nightmare frequency $(d=1.7)$ whereas there was no change in the control group. However, since the control group received no treatment, it is impossible to determine whether the improvement is due to the placebo effect. However, there was no significant difference between the groups in PTSD symptoms ( $d=0.22$ for the treatment group and 0.27 for the control group).

St. Onge, Mercier, and De Konick (2009) extended IRT research even further by utilizing the treatment in a sample of 20 children age $9-11$ with moderate to severe nightmares without PTSD. The researchers advertised for the study by posting and distributing 18,000 flyers. From 
those fliers the researchers received 109 phone calls from interested participants of which 50 (46\%) of the children met criteria. Of these 50, 24 chose not to participate, and six dropped out during the study, leaving a sample of 20. The inclusion criteria were that the participant was between 9 and 11 and had one or more nightmare per week for six months or more. Participants were excluded if they took medications that affect sleep, had trauma-related nightmares, or had co-morbid affective conditions or PTSD. The children were randomly assigned to IRT $(\mathrm{n}=9)$ or waitlist control $(n=11)$. The intervention consisted of an initial meeting, a one-session treatment with unspecified homework four weeks later, and two follow-up sessions (eight weeks and nine months). The authors found that nightmare frequency was significantly reduced in the IRT group when compared with the waitlist control group, and this difference was maintained over nine months.

Forbes and colleagues (2003) were the first to study IRT in a veteran sample. Their sample consisted of 12 Australian Vietnam veterans who had been treated for PTSD in the past but still were having at least one trauma-related nightmare per week. The 12 participants took part in six weekly 90 minute group sessions and then were assessed at post-treatment, after three months, and after one year. At post-treatment there were significant reductions in nightmare frequency $(d=0.70)$, nightmare intensity $(d=0.55)$, depression symptoms $(d=0.43)$, and anxiety symptoms $(d=0.20)$. All of the significant treatment gains were still present at the year follow-up showing that IRT had a lasting impact in this sample. However, due to the absence of a control group, it is impossible to determine whether IRT was effective beyond placebo effect.

In the only study utilizing polysomnography to investigate IRT, Germain and Nielsen (2003) examined the impact of one session of imagery rehearsal therapy on nightmare frequency, psychological distress, and sleep quality (measured by polysomnography) in an uncontrolled 
study. Participants had to be at least 18 years of age and had to report having one nightmare per week for at least 6 months. IRT was conducted in a small group format over one session. At follow-up approximately two months post-treatment, participants had significantly fewer nightmares $(d=1.06)$ and significantly less anxiety symptoms $(d=1.01)$ than prior to treatment. However, reductions in depressive symptoms $(d=0.79)$ and nightmare distress $(d=0.9)$ failed to reach significance. Also, due to the lack of a comparison group, it is unclear whether the reduction in nightmares was due to IRT or a placebo effect. Polysomnography revealed no significant difference in the percent of sleep that was REM sleep, REM latency, or REM density post-treatment.

Nappi and colleagues (2010) extended the work done by Forbes and colleagues (2003) by testing imagery rehearsal therapy in a larger sample of 58 veterans. Imagery rehearsal therapy was given over the course of 5 sessions. However, it should be noted that IRT was not administered in a standardized way and was given in both individual and group format. Nonetheless, at the end of treatment there was a significant decrease in nightmare frequency $(d=$ 0.45), nightmare severity $(d=0.81)$, insomnia symptoms $(d=0.72)$, and PTSD symptoms $(d=$ 1.03). However, since a control group was not utilized, one cannot determine whether IRT was effective beyond the placebo effect.

In sum, there is a growing literature suggesting that imagery rehearsal therapy is effective in treating nightmares. Further, several studies suggest that treating nightmares with imagery rehearsal therapy may also improve sleep quality, and reduce symptoms of insomnia, depression, anxiety, and PTSD. It has been shown to reduce nightmare frequency (and in some studies, severity) in children, adolescents, adults, and veterans. Although the literature on IRT is substantial, much of the supporting studies are uncontrolled. Similar to exposure therapy, IRT 
requires daily practice, which may affect compliance. It is also not clear that IRT is a more effective treatment than recording one's nightmares, which may be a form of exposure. It is also unclear whether the reduction in psychopathology (i.e. symptoms of depression, anxiety, and PTSD) precedes or succeeds a reduction in nightmares. Thus, research has yet to determine whether the reduction in nightmares leads to reductions in symptoms of psychopathology, or vice versa.

Research investigating whether treating nightmares using IRT reduces suicidal behaviors is needed. Nightmares have been found to be related to suicidal ideation (Nadorff, et al., 2011), and predict future suicide attempts (Sjöström, et al., 2009) and death by suicide (Tanskanen, et al., 2001). It is possible that reducing nightmares would also reduce suicidal ideation and make an individual less likely to attempt or die by suicide in the future. Imagery rehearsal therapy seems like a logical choice to treat nightmares among individuals who have been hospitalized due to suicide risk as research has demonstrated that it can be effectively administered in one session (Krakow, Kellner, Pathak, \& Lambert 1996), and hence can be conducted during a short hospitalization. However, Barry Krakow, the author of the treatment, has suggested that one’s nightmares may get worse shortly after receiving IRT due to the client focusing on their nightmares and the distress they cause as a part of treatment, and hence it may not be appropriate for suicidal individuals (Talbot, 2009). Although there are no data showing that nightmares get worse after receiving IRT, primarily due to the lack of studies utilizing continuous measures of nightmares, it would be inappropriate to test IRT on individuals at risk of suicide until it is known if IRT results in a brief increase in nightmares, and if so, if this increase is related to higher levels of depressive symptoms and suicidal ideation. 


\section{Statement of the Problem}

Nightmares are common, with approximately 2-6\% of the adult population likely suffering from a nightmare disorder (Levin \& Nielsen, 2007). This fact is significant not only because of the distress caused by nightmares, but also because nightmares have been linked with several other mental health disorders, such as PTSD (American Psychiatric Association, 2000).

Imagery rehearsal therapy has shown promise not only for treatment of nightmares but also potentially for treating other disorders. Research has demonstrated that IRT significantly reduces not only nightmare frequency but also symptoms of anxiety, depression, and PTSD (Krakow, Johnson, et al. 2001). However, few studies have evaluated the effects of conducting IRT using a single session format. The studies that have been conducted utilizing a one session treatment failed to measure nightmare severity in addition to nightmare frequency. A study examining the impact of one session of imagery rehearsal therapy on nightmare severity would significantly add to the literature and would support the use of imagery rehearsal therapy as a one session treatment.

A significant limitation of all of the studies examining imagery rehearsal therapy to date is they have not examined nightmares continuously following treatment. Instead, they followed up with participants several months later. A study that utilizes continuous measurement of nightmares would significantly add to the literature by enabling researchers to examine how quickly imagery rehearsal therapy reduces nightmares.

The literature on imagery rehearsal therapy is also limited by the fact that no study has examined the temporal precedence of the reduction in nightmares compared with the reduction of the symptoms of psychopathology. Knowing the temporal precedence would help in understanding the mechanism by which nightmares occur, which may help in developing 
treatments for nightmares. Lastly, little research has examined the effects of IRT using an objective measure of sleep. Using a more objective measure to immediately account for a nightmare as soon as it happens would help reduce error caused by inaccurate memories.

The current study aimed to address these limitations through testing imagery rehearsal therapy utilizing a non-concurrent multiple baseline design in a sample of adults. The current study measured nightmare frequency continuously using an immediate report method (actigraphy event marker) and a daily reporting method (online questionnaire). The study also examined nightmare severity continuously through an online questionnaire.

The current project assessed nightmares and mood daily, as well as suicidal ideation at three points, to help determine whether treating nightmares leads to a brief increase in nightmares, depressed mood, and suicidal ideation. If this is the case, IRT may be contraindicated for individuals at risk of suicide.

\section{Present Study}

The present study sought to examine and characterize IRT by utilizing a non-concurrent multiple baseline design. The study had two primary aims: the first aim was to determine whether nightmares are reduced through use of imagery rehearsal therapy and the second aim assessed whether imagery rehearsal therapy results in an increase in nightmares shortly after treatment. Actigraphy was also used throughout the study to help differentiate between sleep and wake in the participants.

Aim 1: To determine whether or not nightmares and disturbing dreams (measured together) are reduced through use of IRT 
Hypothesis 1: There will be a decrease of at least two nightmares and disturbing dreams per week between baseline and the end of the follow-up period after treatment with Imagery Rehearsal Therapy.

Hypothesis 2: There will be a decrease in nightmare and disturbing dream severity between baseline and the end of the follow-up period after treatment with Imagery Rehearsal Therapy.

Hypothesis 3: Participants will have fewer nocturnal awakenings of 10 minutes or longer as of the end of the follow-up period when compared to baseline.

Hypothesis 4: Participants will have higher sleep efficiency as of the end of the follow-up period when compared to baseline.

Aim 2: To determine whether or not IRT results in a temporary increase in nightmares following treatment.

Hypothesis 5: There will be an increase in nightmare and disturbing dream frequency in the week following treatment when compared with baseline.

Hypothesis 6: There will be an increase in nightmare and disturbing dream severity in the week following treatment when compared with baseline.

Hypothesis 7: There will be an increase in negative affect in the week following treatment when compared with baseline.

The study also had an exploratory aim: to characterize the nightmares experienced by participants. To characterize the nightmares experienced by participants, the investigator asked 
participants to describe their nightmares in the initial study session and then conducted a directed content analysis of the descriptions.

\section{Method}

\section{Participants}

Participants were recruited from the community through online, television, and Personal Rapid Transit system (PRT) advertisements, as well as newspaper stories. The inclusion criteria required participants to report, on average, at least three nightmares per week for at least the last six months and meet the ICSD-2 criteria for nightmare disorder (American Academy of Sleep Medicine, 2006), to be included in the study. Requiring three nightmares per week, on average, is higher than the cutoff for other nightmare treatment studies. However, the higher threshold was selected to attempt to avoid floor effects, and to be consistent with the mean nightmare frequency at baseline found in other studies. Participants were excluded from the study if they reported taking any medications that may cause nightmares, reported current suicidal ideation, or if they reported that they were currently abusing drugs or alcohol. Participants who completed the study received $\$ 300$ compensation for their time and effort.

In total, 42 individuals contacted the researchers inquiring about participation in the study (see Figure 1). Of these, 14 passed the initial screening (assessing whether the individual reported, on average, three nightmares per week or more) and were invited to come in for a screening session. Two of these individuals failed to come to the initial appointment, leaving 12 participants. A total of six participants met study criteria and were invited into the study. However, one participant declined participation due to not wanting to wear the actigraph, and the other had a disconnected phone and did not respond to emails from the research team, resulting in four participants who successfully entered and subsequently completed the study. Participant 
001 was a 50 year old caucasian female. She had completed an associate’s degree and was in a committed relationship. Participant 008 was a 22 year old Hispanic female. She had completed some college and was in a committed relationship. Participant 010 was a 42 year old caucasian female. She had completed an advanced degree and was in a committed relationship. Lastly, Participant 012 was a 23 year old caucasian female. She had completed a bachelor's degree and was in a committed relationship.. The study was conducted in accordance with the IRB protocol and APA ethical guidelines. Informed consent was obtained from all participants.

\section{Measures}

Actigraphy. The participants' sleep and wake periods were objectively estimated for the duration of the study utilizing an Actiwatch-64 (AW-64) actigraph (Philips-Respironics Mini Mitter). Actigraphy has been validated against polysomnography, which is considered the gold standard for measuring sleep (de Souza et al., 2003; Jean-Louis et al., 1996; Sadeh \& Acebo, 2002). The actigraph estimates sleep and wake periods by detecting a participant's movement over an epoch, or specified time period. The current study utilized 30-second epochs, which allowed the actigraph to store 22 days of data. The AW-64 is equipped with an event marker button, which the participants pushed whenever they went to bed, woke up in the morning, or had a nightmare. The number of nightmares was determined by subtracting two (one for bed time and one for wake time) from the total number of event marks, with the remainder being nightmares. Nocturnal awakenings were identified by an Excel macro that identified times when there were 20 consecutive epochs (for a total of 10 minutes) of wake following sleep. The data were analyzed using Actiware Software Version 5.4.

Daily Nightmare Ratings. To date there is no validated measure designed to assess nightmares daily. However, several previous studies investigating nightmare treatments have 
had participants record their nightmare frequency and severity daily (Burgess, et al., 1998;

Cellucci \& Lawrence, 1978; Forbes, et al., 2003; Grandi, et al., 2006; Nappi, et al., 2010). The current study assessed daily nightmare frequency in a similar way. Participants were asked to press the event marker on the actigraph whenever they had a nightmare. In addition, participants logged in to a secure website and report how many nightmares they had during the previous night. Participants rated the severity of their nightmares for the night on a 0 to 4 Likert-style scale $(0$ = not distressing -4 = very distressing $)$.

Beck Anxiety Inventory (BAI; Beck \& Steer1993a). The BAI is a 21-item self-report measure that has been validated to measure anxiety symptoms over the last week. Each item is scored on a 0 to 3 scale with total scores for the scale ranging from 0 to 63 . Scores of $0-7$ indicate minimal levels of anxiety symptoms, 8 - 15 indicate mild anxiety symptoms, 16 - 25 indicate moderate anxiety symptoms, and 26 or greater indicate severe anxiety symptoms. The BAI has high internal consistency with an alpha of 0.92 and test-retest reliability of 0.75 (Beck \& Steer1993a). The BAI was collected during the baseline session, treatment session, and final session.

\section{Beck Depression Inventory-Second Edition (BDI-II; Beck, Steer, \& Brown, 1996).}

The BDI-II is a 21-item self-report measure that has been validated to measure depressive symptoms over the last two weeks. It was revised from the original Beck Depression Inventory (Beck \& Steer1987) to be in-line with the DSM-IV. Items are scored on a 0 to 3 scale, with a total possible score being 63. Scores from 0 - 13 indicate minimal depressive symptoms, 14 - 19 indicate mild depressive symptoms, 20 - 28 indicate moderate depressive symptoms, and scores of 29 or higher indicate severe depressive symptoms. The BDI has high internal consistency and 
one-week test-retest reliability (Beck, Steer, \& Brown 1996). The BDI was collected during the baseline session, treatment session, and final session.

Beck Hopelessness Scale (BHS; Beck \& Steer1993b). The BHS is a 20-item, true-false measure of hopelessness. It was designed to assess three components of hopelessness: feelings about the future, loss of motivation, and expectations. It has acceptable internal consistency and test-retest reliability. Research suggests that the BHS may be helpful for identifying individuals who are at risk of dying by suicide. In a sample of psychiatric outpatients, 16 of the 17 (94\%) of those who died by suicide had BHS scores of 9 or higher (Beck, Brown, Berchick, \& Stewart, 1990). The BHS was collected during the baseline session, treatment session, and final session.

Beck Scale for Suicide Ideation (BSS; Beck \& Steer 1993c). The BSS is a 21-item self-report measure that has been validated as a measure of suicidal thoughts and plans over the past week. It was used in the current study to screen out individuals with current suicidal ideation. The items are scored on a three-point (0 - 2) Likert-type scale, although only 19 of the items are scored, yielding total scores ranging from 0 to 38. The two un-scored items assess the number of previous suicide attempts and the suicide intent in the last attempt. The BSS has high internal reliability and moderate test-retest validity (Beck \& Steer, 1993c). The BSS was collected during the baseline session, treatment session, and final session.

Disturbing Dreams and Nightmare Severity Index (DDNSI, Krakow, et al. 2002). The DDNSI is a revised version of the Nightmare Frequency Questionnaire (Krakow, et al., 2000) and was used to measure nightmare severity and frequency. It measures the number of nights with nightmares per week (0-7 nights) and number of total nightmares per week (0-14 nightmares). The DDNSI also measures the severity and intensity of the nightmare on a Likerttype scale ranging from no problem (0) to extremely severe problem (6) as well as how often 
nightmares result in awakenings ranging from never/rarely (0) to always (4). A score greater than 10 may indicate the presence of a nightmare disorder (Krakow, et al., 2002). The DDNSI has shown acceptable reliability ( $a=0.87$ ) in prior studies (Nadorff, et al., 2011). The DDNSI was collected during the baseline session, treatment session, and final session.

Insomnia Severity Index (ISI; Bastien, Vallieres, \& Morin, 2001). The ISI is a 7-item self-report scale that assesses the individual's subjective report of insomnia for the past two weeks. Each item is scored on a 0-4 scale, with total scores ranging from 0-28. Scores of 0-14 are considered to be sub-clinical insomnia or no insomnia, 15-21 are considered moderate insomnia, and 22-28 are considered to be severe insomnia. The ISI has been shown to have adequate test-retest reliability over three months and concurrent validity with sleep diaries and polysomnography, and has been used in previous research as a measure of insomnia severity (Bastien, Vallieres, \& Morin, 2001; Savard, Savard, Simard, \& Ivers, 2005). The ISI has also been used to determine the presence or absence of clinically significant insomnia symptoms using a cutoff of 15 (Bernert, Turvey, Conwell, \& Joiner, 2007; Tang, Wright, \& Salkovskis, 2007). The ISI was collected during the baseline session, treatment session, and final session.

\section{Michigan Assessment-Screening Test for Alcohol and Drugs (MAST/AD;}

Westermeyer, Yargic, \& Thuras, 2004). The MAST/AD is a 24-item yes/no scale that is scored on a weighted scale. It is a revision to the Michigan Alcohol Screening Test (MAST; Selzer, 1971) designed to screen for alcohol and drug abuse and dependence. The only modification to the original MAST was that the term "alcohol” on the MAST was replaced with "alcohol and/or drugs" on the MAST/AD. In the current study, it was used to help identify individuals who are likely abusing or dependent upon drugs or alcohol. Since the only change in the measure is the wording to include drugs in each item in addition to alcohol, a cutoff score of 13, which has been 
established for the MAST (Ross, Gavin, \& Skinner, 1990), was used to identify those who may be at risk of abusing drugs or alcohol. If above cutoff, participants were then asked questions modeled after the DSM criteria for alcohol and substance abuse and dependence, and were excluded if they met criteria for either alcohol or substance use or dependence. The MAST/AD was collected during the baseline session, treatment session, and final session.

Positive and Negative Affect Scales (PANAS; Watson, Clark, \& Tellegen, 1988). The PANAS is a 20-item measure designed to measure positive and negative affect. The PANAS has been used to assess affect in a variety of different time periods ranging from the present moment to the last year (Watson, et al., 1988) and has been used daily in several studies to assess daily mood (Denissen, Butalid, Penke, \& van Aken, 2008; Jones, O'Connor, Conner, McMillan, \& Ferguson, 2007; Roper \& Yorgason, 2009). In the current study, participants completed the PANAS daily online.

The PANAS consists of 20 adjectives (e.g. for positive affect: inspired, attentive, and enthusiastic; for negative affect: distressed, scared, ashamed). Each adjective is rated on a scale from 1 (very slightly or not at all) to 5 (extremely). The PANAS has been shown to be related to distress, depression, and state anxiety. It has been shown to have adequate internal consistency with a Cronbach's alpha of .90 for positive affect and .87 for negative affect when assessing one's affect for that day (Watson, et al., 1988).

PTSD Checklist-Civilian Version (PCL; Weathers, et al., 1993). The PCL is a measure of Posttraumatic stress disorder symptom severity. It consists of 17 questions in which participants are asked if they are bothered by each symptom on a scale ranging from 1 (not at all) to 5 (extremely), with total scores ranging from 17 to 85 (Weathers, et al., 2003). There is no single agreed-upon cutoff for the PCL, but 44 and 50 have each been used as cutoff scores in 
prior research (Ruggiero, Del Ben, Scotti, \& Rabalais, 2003). The PCL has high internal consistency (alpha $=0.94)$ and has been shown to be valid when compared to a clinicianadministered PTSD scale (Peterlin, Tietjen, Meng, Lidicker, \& Bigal, 2008). The PCL has shown acceptable reliability $(a=0.92)$ in prior studies (Nadorff, et al., 2011). The PCL was collected during the baseline session, treatment session, and final session.

Clinician-Administered PTSD Scale (CAPS; Blake, Weathers, Nagy, \& Kaloupek, 1995). The CAPS is a widely-used structured interview designed to help researchers diagnose post-traumatic stress disorder using DSM-IV criteria. It is made up of 30 questions, 17 corresponding with DSM symptoms of PTSD, broken into three clusters: re-experiencing, avoidance/numbing, and hyperarousal. Items are scored on a five-point Likert-style scale ranging from 0 to 4 . A symptom is counted as present if the frequency score is 1 or greater and the severity is 2 or greater. By looking at the symptoms endorsed, the CAPS interview can be used to diagnose current and lifetime PTSD according to DSM-IV criteria (Blake, et al., 1995). The CAPS interview was administered to assess current PTSD during the baseline and final session.

Qualitative Nightmare Questions: Open-ended questions about the experience of having nightmares were developed for this study for the qualitative aim (see Appendix 10). These questions asked participants to describe their nightmares, the effect nightmares have on their lives, how their nightmares have changed over time, and their discussions about nightmares with their healthcare providers. The qualitative questions were administered during the baseline session.

\section{Procedure}


Four participants were recruited from the community through a story in the campus newspaper, advertisements on public access TV, an email notification to faculty and staff at the host institution, and advertisements on the PRT screens. Participants who were interested in the study and passed the initial phone screening (assessing whether the individual reported, on average, three nightmares per week or more) attended an individual baseline session at the Quin Curtis Center for Psychological Services at West Virginia University. During this session, the study was explained and informed consent was obtained from the participant. The participants then were administered the CAPS structured clinical interview, to assess for PTSD, and completed the ISI, DDNSI, BDI, BAI, BHS, BSS, and PCL. The researcher then conducted an unstructured clinical interview to assess the participant's nightmares in order to determine if the participant had, on average, at least three nightmares per week over the last six months and met criteria for nightmare disorder based upon ICSD-2 criteria (American Academy of Sleep Medicine, 2006). The researcher also assessed for current alcohol or drug abuse and current suicidal ideation during the unstructured interview. Individuals not meeting criteria for nightmare disorder, with current suicidal ideation (defined as a score $>0$ on items $4-5$ on the BSS), or who were currently abusing alcohol or drugs (defined as a MAST/AD score over 12 and meeting criteria for alcohol or substance use or dependence) were excluded from the study. The remaining participants were asked to describe their nightmares and to talk about what it is like to have nightmares (see appendix 10 for questions). A directed content analysis was then conducted to analyze the qualitative information. The qualitative information was videotaped and transcribed under each question. Then, salient expressions made by each participant were identified and synthesized. 
Following the initial session, the participants were contacted to let them know whether or not they met the study's inclusion criteria. The participants who met the inclusion criteria then came back to the clinic so they could be trained on how to login to a website so they could complete the PANAS and nightmare questions daily, as well as be given an actigraph.

\section{Design}

The study utilized a non-concurrent multiple baseline design. Similar to a concurrent design, the treatment sessions were staggered, but participants started the study at different times. Non-concurrent multiple baseline designs have several strengths. First, they do not require the withdrawal of treatment, which would be impossible in IRT since IRT involves learning skills that cannot be withdrawn. Second, the effect is replicated, strengthening the likelihood that the effect is due to the treatment and not confounding factors. However, non-concurrent multiple baseline designs also have limitations. First, although one can demonstrate an association with temporal precedence between an independent and dependent variable, it is not a causal design. It also requires that a stable baseline be established prior to treatment, which is a limitation as it may delay the administration of treatment. Third, confounding effects, such as period effects, may exist since the participants did not begin at the same time. Lastly, a multiple baseline design requires frequent measurement, which may be taxing on the participant. Despite these limitations, a multiple baseline design is a strong study design that enabled the study of IRT in a more detailed way than had been done previously.

Baseline began for each participant as soon as they were able to come in and receive the actigraph following the initial session. Daily nightmare frequency and severity ratings were aggregated into weekly scores. Participants remained in baseline until a stable nightmare frequency baseline was established (no negative trend in the last three weeks). Once the baseline 
was stable, the researcher contacted the participant to schedule the therapy session.

Occasionally, there were a few days between when the participant reached stability and when they were able to come in for a treatment session $(M=2.25$, range $0-5)$. However, the participants' nightmare trends were either stable or increasing over this brief period, so treatment was still indicated.

Prior to the treatment session, the participants once again completed the ISI, DDNSI, BDI, BAI, BHS, BSS, and PCL. No participants reported current suicidal ideation (defined as a score $>0$ on items 4-5 on the BSS), but should they have done so, they would have been referred for depression treatment and would receive nightmare treatment at a later time. The session began with psycho-education about nightmares. Participants were told that nightmares are “a learned behavior” (Krakow, 2002, p. 18) and that behaviors can be un-learned. Participants also received information about sleep hygiene as part of the psycho-education. The researcher then discussed the causes and course of nightmares, including how nightmares are thought to be a “learned behavior” (Krakow, 2002, p. 18). The researcher then presented the process of changing one's nightmares and rehearsing the new dream. Lastly, the participant and researcher discussed how to overcome or avoid potential obstacles that may prevent the participant from having a positive outcome.

A follow-up session was scheduled with participants one month after treatment. During this session, a researcher administered the CAPS structured interview and asked the participant to complete the ISI, DDNSI, BDI, BAI, BHS, and PCL. Appropriate referrals were available for participants if required. 


\section{Results}

Participants were in the baseline condition until their last three weeks showed a positive trend (range 3 - 8 weeks). Should a participant reach stability during the same week that another had received treatment, the participant remained in the baseline condition until they were stable during a week in which no one had received treatment. Thus, no two participants received treatment during the same week. All treatment sessions were rated using a checklist to ensure that the treatment provided was faithful to IRT. All sessions were rated as having strong fidelity, indicating that all components of IRT were adequately covered. Participants were asked whether they practiced their new dream every day as part of the daily online questionnaire. Overall, participants reported practicing during $95.7 \%$ of the days following treatment, practicing on average 1.75 times per day $(S D=0.62)$.

Regression coefficients for nightmare frequency assessed by the event marker were calculated for the entire baseline, as well as the last three weeks of baseline, for every participant. The regression coefficient for the entire baseline for participant 001 was $b=0$, for participant 008 was $b=-0.09$, for participant 010 was $b=0.02$, and for participant 012 was $b=$ 0.04. For the last three weeks of baseline, the regression coefficient for participant 001 was $b=$ 0 , for participant 008 was $b=0.14$, for participant 010 was $b=0$, and for participant 012 was $b=$ 0.14 .

The data for the study were analyzed using visual inspection for each participant. Following the analysis of each graph, an overall conclusion was made based upon the data from all four participants.

We first evaluated whether or not nightmares and disturbing dreams (measured together) are reduced following IRT. The first hypothesis stated that there would be a decrease of at least 
two nightmares and disturbing dreams per week between baseline and the end of the follow-up period after treatment with Imagery Rehearsal Therapy. First, the results for nightmare frequency measured by the event marker will be presented, as this was the primary measure, followed by nightmare frequency as measured by an online questionnaire (see Figure 2 .

Participant 001 showed a weak treatment effect on level with no change in trend. Participant 008 showed no treatment effect. Participant 010 demonstrated a weak treatment effect with a possible change in level, although the reduction was less than two nightmares per week. Lastly, participant 012 showed no clear change in trend or level. Overall, there were no standout effects. However, there were minor initial reductions in nightmare frequency for participants 001, 010, and 012, where the participant's first point after treatment was lower than the last two points of baseline. Overall, based upon nightmare frequency as measured by the actigraph’s event marker, the first hypothesis was not supported.

The online questionnaire nightmare frequency, an alternate measure of nightmare frequency, was also examined. For participant 001, the overall trend was negative, making it difficult to determine whether there was an effect. However, there appears to be a weak change in level. Participant 008 had no change in level but a change in trend. Participant 010 had no change in level or trend, but did have a change in variability, with substantially less variability following treatment when compared to baseline. Lastly, participant 012 showed a change in trend, but no change in level. Overall, only one participant showed a weak change in level, although two demonstrated a change in trend. Thus, similar to the nightmare frequency as measured by event marker analyses, there was not a substantial change in nightmare frequency following treatment as measured by an online questionnaire. 
Next we examined what occured immediately following nightmare treatment. Although treatment was expected to be associated with a decrease in nightmares and disturbing dreams, one objective of the project was to evaluate whether there is a temporary increase in nightmares and disturbing dream frequency, severity, and negative affect in the week following treatment.

It was also postulated that there will be an increase in nightmare and disturbing dream frequency in the week following treatment when compared with baseline (see Figure 2). Examining the nightmare frequency data from the event marker, three of the four participants (001, 008, and 012) had a reduction in nightmare frequency in the week following baseline when compared to the last week of baseline, while 010 had stable nightmare frequency. When compared to the entire baseline, participant 001 had a weak negative change in level, and the other participants were stable. The online questionnaire nightmare frequency data paint a similar picture, with all four participants having a decrease in nightmares during the week following treatment when compared with the last week of baseline. When the first week post-treatment was compared with the overall baseline, none of the participants demonstrated a change in level. Thus, the fifth hypothesis was not supported as nightmare frequency did not increase in the first week after treatment on either event marker or online questionnaire.

We also predicted that there would be a decrease in nightmare and disturbing dream severity between baseline and the end of the follow-up period after treatment with IRT (see Figure 5). Participant 001 again had a negative trend, making it difficult to show a treatment effect, with a possible weak change in level. Participant 008 showed a weak change in trend, but no change in level. Participant 010 showed a weak and transient change in level, as nightmare severity decreased immediately after treatment, but then began to trend positively again. Lastly, 
participant 012 had a possible change in trend, but no change in level. Overall, hypothesis two was not supported, as the changes on trend and level were weak and mixed.

It was also anticipated that there would be an increase in nightmare and disturbing dream severity in the week following treatment when compared with baseline (see Figure 5).

Examining the nightmare severity data, all four participants had a reduction in nightmare severity in the week following baseline when compared to the last week of baseline. When compared to the entire baseline, none of the participants had a change in level. Thus, the sixth hypothesis was not supported, as nightmare severity did not increase in the first week after treatment.

Hypothesis three stated that participants would have fewer nocturnal awakenings of 10 minutes or longer post-treatment when compared to baseline (see Figure 6). For participant 001, there was a positive change in level, with potential change in trend. Participant 008 demonstrated an increase in variability following treatment, but no clear change in trend or level. Participant 010 was stable with no change in level or trend. Lastly, participant 012 had a stable trend, but a positive change in level. Overall, the third hypothesis was not supported, as none of the participants had a reduction in nocturnal awakenings. In fact, two of the four participants showed an increase in nocturnal awakenings. It appears that the increases are idiosyncratic, and when they do occur, they often result in a change in level with no substantial effect on sleep efficiency.

The fourth hypothesis postulated that participants would have higher sleep efficiency at of the end of the follow-up period when compared to baseline (see Figure 7). For all four participants, there was no change in level or trend, indicating a lack of an effect. Therefore, the fourth hypothesis was not supported. 
The last hypothesis is that there would be an increase in negative affect in the week following treatment when compared with baseline (see Figure 8). In the week following treatment, participant 001's negative affect was substantially higher than the last week of baseline, and was slightly higher than the overall baseline, indicating a weak change in level. Participant 008 had a slight decrease in negative affect in the week following treatment, with no overall change in level when compared to the entire baseline. Participant 010 had a small decrease in negative affect when compared to the last week of baseline, and overall a small negative change in level. Lastly, participant 012 had a slight increase in negative affect in the week following treatment when compared to the last week of baseline, but when compared to the overall baseline there was no change in level. Overall, the immediate effect of treatment on negative affect appears to be idiosyncratic. Looking first at the trend, there was an increase in trend in half of the cases, and a decrease in the other half. The findings were also mixed in relation to level: there was a weak increase in level in one case, weak decrease in level in one case and no change in level in the other two. Due to the lack of consistent findings suggesting that negative affect increases immediately following treatment, the seventh hypothesis was not supported.

The study also had an exploratory aim to characterize the nightmares experienced by participants through directed content analysis. For the first question, where participants were asked to describe the nightmares they had most often, the participants reported few recurrent dreams or dreams that were replays of actual events. Instead, the dreams were often described as being strange, or unrealistic. Three of the four participants described having nightmares that were based on topics they were anxious about, such as relationships or school. Question two asked the participants to describe the effect that nightmares had on their lives. Two participants 
reported that following a nightmare it was difficult to figure out what is real and what was part of the dream, and it took some time to get back to reality. Relatedly, a third participant reported feeling anger at her significant other due to negative dreams about him. The fourth participant reported that her nightmares often led her to avoid sleep, which she felt increased her depression and anxiety symptoms. Participants were also asked to describe how they felt immediately after having a nightmare. Half of the participants reported waking up sweating, with their heart racing, and two reported feeling very confused about what reality is. Further, two of the participants reported feeling tired or fatigued after having a nightmare. Thus, all participants reported that nightmares were negative experiences.

Questions three, five and six examined how the participants’ nightmares have evolved over time, as well as what they believe may lead to having nightmares. The participants reported that they believed that their past experiences and current situations have significantly contributed to the nightmares. Three of the four participants reported that prior experiences or stressful events have contributed to their nightmare problems, and the fourth participant believed that her current situation was the greatest contributor to her problems. The nightmares participants experienced also seem to have changed over time in both content and intensity. For two of the participants, they reported having nightmares that were not based on reality when they were younger (e.g. being chased by a wolf) but now having nightmares involving people they actually know or real events. All four participants reported more recently having nightmares involving people they knew or actual events in their life. Intensity was idiosyncratic, with one participant reporting a decrease in intensity over time, and one reporting an increase in intensity over the years. As far as the events that participants believed may make it likely that they would have a nightmare that night, two participants reported that feeling stressed, putting things off, or having 
bad things happen during the day would trigger nightmares. However, not everyone reported that they knew what triggered their nightmares, as the other two participants said they were unsure what led to them have nightmares.

The last two questions asked participants what they felt was the most important aspect of having nightmares, as well as whether or not they have reported their nightmares to health care providers. Two of the four participants reported that the most important aspect of having nightmares to them was wondering why they had them, and what the dreams may mean. The other two participants focused on the way that nightmares affect them, one discussing avoiding sleep, and the other reporting feelings of dread. Despite the negative effects of nightmares, and the questions the participants have about having nightmares, only two of the four participants reported having discussed nightmares with health care providers, with both of those disclosures being to psychotherapists. It is not clear whether or not those discussions were prompted by the participant or by the therapist.

The results from the exploratory questionnaires, as well as questionnaires utilized for inclusion/exclusion criteria, can be found in Table 1. These data are included to characterize the sample.

\section{Discussion}

The present study is the first to examine the effects of Imagery Rehearsal Therapy continuously, which allows the current study to explore the mechanisms of change that had previously been ignored by research. It was also strengthened through its use of a single-subject design, which is ideal for examining intra-individual change over time. There was not a substantial decrease in nightmare frequency or severity following treatment with IRT. However, there are several potentially notable findings. First, the finding that nightmare frequency and 
severity did not increase in the week following treatment is clinically significant. Prior to this study, clinicians were urged not to use Imagery Rehearsal Therapy with individuals who are at risk of suicide for fear that the therapy may increase nightmare frequency and severity, leading to an increase in suicide risk (Talbot, 2009). However, given the lack of a treatment effect, it is possible that the treatment was inert, explaining why there was no change in nightmare frequency and severity. Additionally, although we did not consistently find an increase in negative affect following treatment, some participants did show an increase, suggesting that IRT's effect on negative affect may be idiosyncratic. Thus, more research is needed to investigate whether IRT leads to temporary increases in nightmare frequency, severity, and negative affect.

The current study failed to replicate prior findings that IRT leads to a substantial reduction in nightmare frequency and severity. Although, on average, there appears to have been a decrease in nightmare frequency and severity, the amount of variability masks any treatment effects. Further, on secondary measures (i.e. measures that did not require stability prior to treatment), the presence of a negative trend in some graphs made it impossible to determine whether a change was due to treatment or the continuation of a negative trend.

There may be several possible reasons for these negative findings. This failure to replicate may be due to the short time to follow-up when compared to other studies investigating IRT. Most IRT studies follow participants for three months or longer post-treatment (Krakow, Hollifield, et al., 2001; Krakow, et al., 2000; Krakow, Johnston, et al., 2001; Krakow, et al., 1993; Krakow, et al., 1996; Krakow, Sandoval, et al., 2001), whereas the current study only followed participants for four weeks post-treatment. The shorter follow-up period was chosen because the present study required daily monitoring of nightmares and wearing an actigraph, and 
hence a longer follow-up period would have significantly increased participant burden. Further, a literature review revealed no research that suggested that IRT would require more than a month to show a clinically-significant effect. Given that research has shown that the effects of IRT improve from three months post-treatment to six-months post-treatment (Krakow, et al., 1996), it is very possible that the study's follow-up was too soon to see a substantial treatment effect, as the effect may grow over time. It is also possible that a one session treatment with no booster sessions is not a strong enough intervention to see a substantial reduction in nightmare frequency.

The current study significantly adds to the literature by qualitatively examining the experience of having nightmares. This examination not only gives us a glimpse into what it is like to have nightmares, but also has implications for how we treat nightmares. Through better understanding the nightmare experience, researchers can design new treatments. Further, since no two patients are alike, through better understanding the antecedents and consequences of nightmares we can better tailor our interventions to each client. Participants reported that their nightmares were mostly strange or unrealistic, instead of being replays of traumatic events. This may be due to participants having idiopathic nightmares, as none of them met criteria for PTSD based upon the CAPS interview. It was also interesting that several participants reported that their nightmares occurred more often during times that they were anxious or stressed, and that the content of the nightmare was often about things they were anxious or stressed about. This finding suggests that nightmares may be associated with anxiety, which should be investigated in future research. It also has treatment implications. Given that our participants reported that nightmares are often tied with anxiety and/or stress, they may benefit from learning relaxation and stress management skills. 
Looking at the negative effects of nightmares, participants reported both physical and psychological effects. Physically, two participants reported waking up sweating with their heart racing, and two more reported feeling fatigued after having a nightmare. Psychologically, participants reported being confused, angry at others, and were left questioning what is real and what was just a dream. Further, one participant reported that she avoided sleep, especially after a nightmare, for fear of having the same dream again. The severity of the nightmare experience was striking. One participant reported that she often had to call her significant other in another state to calm down enough to return to sleep, and a second participant reported that she would often avoid sleep due to fear of having a nightmare. Thus, it is easy to see how nightmares can significantly affect one’s sleep, and hence one’s quality of life.

Although participants reported that nightmares had a significant negative effect on their life, and they also reported having questions about the reasons for and meaning of their nightmares, only two of the participants had mentioned having nightmares to a health care professional, and none reported having received treatment for nightmares in the past. This suggests that both nightmare sufferers and clinicians may be unaware that nightmares have negative consequences, or that treatment options exist. Given the numerous negative consequences of nightmares, and their association with various psychiatric disorders, it is important for health care providers to identify individuals with nightmares and refer them for proper treatment.

\section{Limitations}

Although the use of a multiple baseline design is one of this study’s greatest strengths, it also has some limitations. First, due to requiring a stable baseline and multiple measurements, multiple baseline designs are not ideal for treatments that take a long time to show an effect. The 
results of this study suggest that IRT may take more than a month to show an effect, and hence, the current study was limited by its relatively short time to follow-up of four weeks. Although this study was limited by the short follow-up, it also adds to the literature by suggesting that IRT may take longer than four weeks to see a substantial reduction in symptoms. The current study was also limited by an unexpectedly high degree of variability in nightmare frequency and severity, which made it difficult to find an effect using multiple baseline design. Utilizing longer aggregation periods, such as two weeks or a month instead of one week, would reduce the negative effect of the variability on the design and make the design more appropriate for studying nightmares. Lastly, the multiple baseline design employed did not allow for us to control for the placebo effect. This could have been done by either utilizing a group design, or a ABAC design where $\mathrm{B}$ is a placebo treatment and $\mathrm{C}$ is the treatment of interest.

However, despite being one of the weakest of the single-subject designs, the nonconcurrent baseline design has several advantages over group designs. Primarily, multiple baseline designs are ideal for looking at change within individuals over several time points, which is not suited to group designs. Since the goal of the study was to look at daily change within individuals, the multiple baseline design was ideal for examining whether nightmare frequency, severity, and negative affect increased shortly after treatment.

Although treatment adherence was rated strongly in the present study, we failed to see a substantial reduction in nightmare frequency or severity. This may be due to participants not understanding the treatment, even though it was presented properly. Future research should incorporate measures of understanding to ensure that participants comprehended the treatment.

The generalizability of the study was limited by the small number of participants, as well as the fact that all participants were female and had idiopathic nightmares. However, these 
limitations fit well with the multiple baseline design, which works best when as many potential confounds are controlled as possible.

\section{Conclusions and Future Directions}

Although the present study failed to show a treatment effect for IRT on nightmare frequency and severity, it adds to the literature by demonstrating that in this sample nightmare frequency, severity, and negative affect did not increase in the week following IRT. This is important as the treatment's author has stated that IRT should not be used with individuals at risk of suicide due to anecdotal evidence that treatment may increase nightmare frequency (Talbot, 2009). However, past research has shown that having nightmares significantly increases the risk of suicide re-attempt (Sjöström, et al., 2009), and as such those at risk of suicide may greatly benefit from nightmare treatment. Further research is needed to examine whether nightmares increase following IRT in a larger sample, and if so, whether or not it leads to an increase in suicidal ideation.

The use of a multiple baseline design was a significant strength of the present study, as it made it possible to examine the participants' responses day-by-day so one could look for increases in nightmares or negative affect immediately following treatment. The results of this study suggest, however, that it may not have been implemented optimally, which may explain the lack of results. First of all, it was extremely difficult to find participants who reported enough nightmares to see an effect with weekly aggregation. Although this could be addressed by aggregating over two weeks, or a month, instead of one week, it would significantly increase the length of the study. Second, it became clear that nightmares are highly variable, which makes it difficult to establish a stable baseline and, hence, conclusions about treatment effects. Aggregating over a longer period of time would have likely reduced the variability in the 
measures. Lastly, the present study suggests that participants may not see a significant benefit from IRT in the first month post-treatment. In order to examine whether there was a treatment effect, one would likely have to significantly lengthen the follow-up period, which would add to participant burden and potentially make the study infeasible. In sum, the utilization of a multiple baseline design was invaluable for assessing whether treatment led to a transient increase in nightmares and negative affect. However, to be used effectively, one should aggregate over a longer period of time, which may reduce the feasibility of using a single-subject design. Future research may want to utilize a mixed design, where the study utilizes a multiple baseline design which ends shortly after treatment, but the study continues to follow the participants for three to six months post-treatment.

In conclusion, although the present study failed to replicate the findings of other studies that nightmare frequency and severity are reduced by IRT, it does provide evidence that nightmare frequency and severity do not increase immediately following treatment. It is hoped that this study lay the groundwork for future studies examining whether nightmare treatment, such as IRT, may be beneficial for individuals at risk of suicide. The current study also significantly adds to the literature by qualitatively examining the nightmare experience, which is an important aspect of the nightmare experience that has not been examined in previous studies. 


\section{References}

Agargun, M. Y., Besiroglu, L., Cilli, A. S., Gulec, M., Aydin, A., Inci, R., \& Selvi, Y. (2007). Nightmares, suicide attempts, and melancholic features in patients with unipolar major depression. Journal of Affective Disorders, 98(3), 267-270.

Agargun, M. Y., Kara, H., Ozer, O. A., Selvi, Y., Kiran, U., \& Kiran, S. (2003). Nightmares and dissociative experiences: The key role of childhood traumatic events. Psychiatry and Clinical Neurosciences, 57(2), 139-145.

Agargun, M. Y., Kara, H., Ozer, O. A., Selvi, Y., Kiran, U., \& Ozer, B. (2003). Clinical importance of nightmare disorder in patients with dissociative disorders. Psychiatry and Clinical Neurosciences, 57(6), 575-579.

American Academy of Sleep Medicine. (2001). International classification of sleep disorders, revised: Diagnostic and coding manual. Chicago, Illinois.

American Academy of Sleep Medicine. (2006). International Classification of Sleep Disorders Second Edition. Chicago, IL: American Academy of Sleep Medicine.

American Psychiatric Association. (2000). Diagnostic and Statistical Manual of Mental Disorders (4th ed., text revision ed.). Washington, DC: Author.

Bastien, C. H., Vallieres, A., \& Morin, C. M. (2001). Validation of the Insomnia Severity Index as an outcome measure for insomnia research. Sleep Medicine, 2(4), 297-307.

Beck, A. T., Brown, G., Berchick, R. J., \& Stewart, B. L. (1990). Relationship between hopelessness and ultimate suicide: A replication with psychiatric outpatients. The American Journal of Psychiatry, 147(2), 190-195.

Beck, A. T., \& Steer, R. A. (1987). Manual for the revised Beck Depression Inventory. San Antonio, TX: Psychological Corporation. 
Beck, A. T., \& Steer, R. A. (1993a). Manual for the Beck Anxiety Inventory. San Antonio, TX: The Psychological Corporation.

Beck, A. T., \& Steer, R. A. (1993b). Manual for the Beck Hopelessness Scale. San Antonio, TX: The Psychological Corporation.

Beck, A. T., \& Steer, R. A. (1993c). Manual for the Beck Scale for Suicide Ideation. San Antonio, TX: The Psychological Corporation.

Beck, A. T., Steer, R. A., \& Brown, G. K. (1996). The Beck Depression Inventory - Second Edition. San Antonio, TX: The Psychological Corporation.

Bernert, R., Turvey, C., Conwell, Y., \& Joiner, T. (2007). Sleep disturbance as a unique risk factor for completed suicide. SLEEP, 30, A344.

Besiroglu, L., Agargun, M. Y., \& Inci, R. (2005). Nightmares and terminal insomnia in depressed patients with and without melancholic features. Psychiatry Research, 133(2-3), 285-287.

Blake, D. D., Weathers, F. W., Nagy, L. M., \& Kaloupek, D. G. (1995). The development of a Clinician-Administered PTSD Scale. Journal of Traumatic Stress, 8(1), 75-90.

Burgess, M., Gill, M., \& Marks, I. (1998). Postal self-exposure treatment of recurrent nightmares: Randomised controlled trial. British Journal of Psychiatry, 172, 257-262.

Cartwright, R. (2001). Crisis Dreaming: Using Your Dreams to Solve Your Problems: IUniverse.

Cellucci, A. J., \& Lawrence, P. S. (1978). The efficacy of systematic desensitization in reducing nightmares. Journal of Behavior Therapy and Experimental Psychiatry, 9(2), 109-114.

Claridge, G., Davis, C., Bellhouse, M., \& Kaptein, S. (1998). Borderline personality, nightmares, and adverse life events in the risk for eating disorders. Personality and Individual Differences, 25(2), 339-351. 
Cukrowicz, K. C., Otamendi, A., Pinto, J. V., Bernert, R. A., Krakow, B., \& Joiner, T. E., Jr. (2006). The impact of insomnia and sleep disturbances on depression and suicidality. Dreaming, 16(1), 1-10.

Davidson, J. R., Hughes, D., Blazer, D. G., \& George, L. K. (1991). Post-traumatic stress disorder in the community: An epidemiological study. Psychological Medicine: A Journal of Research in Psychiatry and the Allied Sciences, 21(3), 713-721.

de Souza, L., Benedita-Silva, A. A. l., Pires, M. L. N., Poyares, D., Tufik, S., \& Calil, H. M. (2003). Further validation of actigraphy for sleep studies. Sleep: Journal of Sleep and Sleep Disorders Research, 26(1), 81-85.

Denissen, J. J. A., Butalid, L., Penke, L., \& van Aken, M. A. G. (2008). The effects of weather on daily mood: A multilevel approach. Emotion, 8(5), 662-667.

Forbes, D., Phelps, A. J., McHugh, A. F., Debenham, P., Hopwood, M., \& Creamer, M. (2003). Imagery rehearsal in the treatment of posttraumatic nightmares in Australian veterans with chronic combat-related PTSD: 12-month follow-up data. Journal Of Traumatic Stress, 16(5), 509-513.

Freud, S. (2004). The Interpretation of Dreams: Kessinger.

Germain, A., \& Nielsen, T. A. (2003). Impact of Imagery Rehearsal Treatment on Distressing Dreams, Psychological Distress, and Sleep Parameters in Nightmare Patients. Behavioral Sleep Medicine, 1(3), 140-154.

Grandi, S., Fabbri, S., Panattoni, N., Gonnella, E., \& Marks, I. (2006). Self-exposure treatment of recurrent nightmares: waiting-list-controlled trial and 4-year follow-up. Psychotherapy And Psychosomatics, 75(6), 384-388. 
Guerrero, J., \& Crocq, M.-A. (1994). Sleep disorders in the elderly: Depression and posttraumatic stress disorder. Journal of Psychosomatic Research, 38(Suppl 1), 141-150.

Hartmann, E. (1981). A preliminary study of the personality of the nightmare sufferer: Relationship to schizophrenia and creativity? The American Journal of Psychiatry, 138(6), 794-797.

Hartmann, E. (1989). Boundaries of dreams, boundaries of dreamers: Thin and thick boundaries as a new personality measure. Psychiatric Journal of the University of Ottawa, 14(4), 557-560.

Hartmann, E. (2001). Dreams and nightmares: the origin and meaning of dreams. New York, NY: Perseus.

Hartmann, E., Russ, D., Oldfield, M., Sivan, I., \& Cooper, S. (1987). Who has nightmares? The personality of the lifelong nightmare sufferer. Archives Of General Psychiatry, 44(1), 4956.

Harvey, A. G., Jones, C., \& Schmidt, D. A. (2003). Sleep and posttraumatic stress disorder: A review. Clinical Psychology Review, 23(3), 377-407.

Herz, M. I., \& Melville, C. (1980). Relapse in schizophrenia. The American Journal of Psychiatry, 137(7), 801-805.

Hobson, J. A., \& McCarley, R. W. (1977). The brain as a dream state generator: An activationsynthesis hypothesis of the dream process. The American Journal of Psychiatry, 134(12), 1335-1348.

Jean-Louis, G., von Gizycki, H., Zizi, F., Fookson, J., Spielman, A., Nunes, J., . . Taub, H. (1996). Determination of sleep and wakefulness with the actigraph data analysis software (ADAS). Sleep, 19(9), 739-743. 
Jones, F., O'Connor, D. B., Conner, M., McMillan, B., \& Ferguson, E. (2007). Impact of daily mood, work hours, and iso-strain variables on self-reported health behaviors. Journal of Applied Psychology, 92(6), 1731-1740.

Kaup, B. A., Ruskin, P. E., \& Nyman, G. (1994). Significant life events and PTSD in elderly World War II veterans. The American Journal of Geriatric Psychiatry, 2(3), 239-243.

Krakow, B. (2002). Turning nightmares into dreams. Albuquerque, New Mexico: Subia Inc. Krakow, B., Hollifield, M., Johnston, L., Koss, M., Schrader, R., Warner, T. D., . . Prince, H. (2001). Imagery rehearsal therapy for chronic nightmares in sexual assault survivors with posttraumatic stress disorder: a randomized controlled trial. JAMA: The Journal Of The American Medical Association, 286(5), 537-545.

Krakow, B., Hollifield, M., Schrader, R., Koss, M., Tandberg, D., Lauriello, J., . . Kellner, R. (2000). A controlled study of imagery rehearsal for chronic nightmares in sexual assault survivors with PTSD: a preliminary report. Journal Of Traumatic Stress, 13(4), 589-609.

Krakow, B., Johnston, L., Melendrez, D., Hollifield, M., Warner, T. D., Chavez-Kennedy, D., \& Herlan, M. J. (2001). An open-label trial of evidence-based cognitive behavior therapy for nightmares and insomnia in crime victims with PTSD. The American Journal of Psychiatry, 158(12), 2043-2047.

Krakow, B., Kellner, R., Neidhardt, J., Pathak, D., \& Lambert, L. (1993). Imagery rehearsal treatment of chronic nightmares: with a thirty month follow-up. Journal Of Behavior Therapy And Experimental Psychiatry, 24(4), 325-330.

Krakow, B., Kellner, R., Pathak, D., \& Lambert, L. (1996). Long term reduction of nightmares with imagery rehearsal treatment. Behavioural and Cognitive Psychotherapy, 24(2), 135148. 
Krakow, B., Melendrez, D. C., Johnston, L. G., Clark, J. O., Santana, E. M., Warner, T. D., .. . Lee, S. A. (2002). Sleep Dynamic Therapy for Cerro Grande Fire evacuees with posttraumatic stress symptoms: a preliminary report. The Journal Of Clinical Psychiatry, 63(8), 673-684.

Krakow, B., Sandoval, D., Schrader, R., Keuhne, B., McBride, L., Yau, C. L., \& Tandberg, D. (2001). Treatment of chronic nightmares in adjudicated adolescent girls in a residential facility. Journal of Adolescent Health, 29(2), 94-100.

Lancee, J., van den Bout, J., \& Spoormaker, V. I. (2010). Expanding self-help Imagery Rehearsal Therapy for nightmares with sleep hygiene and lucid dreaming: A waiting-list controlled trial. International Journal of Dream Research, 3(2), 111-120.

Levin, R. (1998). Nightmares and schizotypy. Psychiatry: Interpersonal and Biological Processes, 61(3), 206-216.

Levin, R., \& Fireman, G. (2002). Nightmare prevalence, nightmare distress, and self-reported psychological disturbance. Sleep: Journal of Sleep and Sleep Disorders Research, 25(2), 205-212.

Levin, R., \& Nielsen, T. (2009). Nightmares, bad dreams, and emotion dysregulation: A review and new neurocognitive model of dreaming. Current Directions in Psychological Science, 18(2), 84-88.

Levin, R., \& Nielsen, T. A. (2007). Disturbed dreaming, posttraumatic stress disorder, and affect distress: A review and neurocognitive model. Psychological Bulletin, 133(3), 482-528.

Mellman, T. A., David, D., Bustamante, V., Torres, J., \& Fins, A. (2001). Dreams in the acute aftermath of trauma and their relationship to PTSD. Journal of Traumatic Stress, 14(1), 241-247. 
Mellman, T. A., David, D., Kulick-Bell, R., \& Hebding, J. (1995). Sleep disturbance and its relationship to psychiatric morbidity after Hurricane Andrew. The American Journal of Psychiatry, 152(11), 1659-1663.

Miller, W. R., \& DiPilato, M. (1983). Treatment of nightmares via relaxation and desensitization: A controlled evaluation. Journal of Consulting and Clinical Psychology, 51(6), 870-877.

Nadorff, M. R., Nazem, S., \& Fiske, A. (2011). Insomnia symptoms, nightmares, and suicidal ideation in a college student sample. SLEEP, 34(1), 93-98.

Nappi, C. M., Drummond, S. P. A., Thorp, S. R., \& McQuaid, J. R. (2010). Effectiveness of imagery rehearsal therapy for the treatment of combat-related nightmares in veterans. Behavior Therapy, 41(2), 237-244.

Neidhardt, E. J., Krakow, B., Kellner, R., \& Pathak, D. (1992). The beneficial effects of one treatment session and recording of nightmares on chronic nightmare sufferers. Sleep: Journal of Sleep Research \& Sleep Medicine, 15(5), 470-473.

Nielsen, T. A., Laberge, L., Paquet, J., Tremblay, R. E., Vitaro, F., \& Montplaisir, J. (2000). Development of disturbing dreams during adolescence and their relation to anxiety symptoms. SLEEP, 23(6), 727-736.

Nielsen, T. A., Stenstrom, P., \& Levin, R. (2006). Nightmare frequency as a function of age, gender, and September 11, 2001: Findings from an Internet questionnaire. Dreaming, 16(3), 145-158.

Ohayon, M. M., \& Shapiro, C. M. (2000). Sleep disturbances and psychiatric disorders associated with posttraumatic stress disorder in the general population. Comprehensive Psychiatry, 41(6), 469-478. 
Paris, J. (2002). Chronic Suicidality Among Patients With Borderline Personality Disorder. Psychiatr Serv, 53(6), 738-742. doi: 10.1176/appi.ps.53.6.738

Peterlin, B. L., Tietjen, G., Meng, S., Lidicker, J., \& Bigal, M. (2008). Post-traumatic stress disorder in episodic and chronic migraine. Headache, 48(4), 517-522.

Purcell, S., Mullington, J., Moffitt, A., \& Hoffmann, R. (1986). Dream self-reflectiveness as a learned cognitive skill. Sleep: Journal of Sleep Research \& Sleep Medicine, 9(3), 423437.

Roper, S. O., \& Yorgason, J. B. (2009). Older adults with diabetes and osteoarthritis and their spouses: Effects of activity limitations, marital happiness, and social contacts on partners' daily mood. Family Relations: An Interdisciplinary Journal of Applied Family Studies, 58(4), 460-474.

Ross, H. E., Gavin, D. R., \& Skinner, H. A. (1990). Diagnostic validity of the MAST and the Alcohol Dependence Scale in the assessment of DSM-III alcohol disorders. Journal of Studies on Alcohol, 51(6), 506-513.

Ruggiero, K. J., Del Ben, K., Scotti, J. R., \& Rabalais, A. E. (2003). Psychometric properties of the PTSD Checklist-Civilian Version. Journal Of Traumatic Stress, 16(5), 495-502.

Sadeh, A., \& Acebo, C. (2002). The role of actigraphy in sleep medicine. Sleep Medicine Reviews, 6(2), 113-124.

Salvio, M.-A., Wood, J. M., Schwartz, J., \& Eichling, P. S. (1992). Nightmare prevalence in the healthy elderly. Psychology and Aging, 7(2), 324-325.

Savard, M.-H. l. n., Savard, J. e., Simard, S. b., \& Ivers, H. (2005). Empirical validation of the Insomnia Severity Index in cancer patients. Psycho-Oncology, 14(6), 429-441. 
Schredl, M., Biemelt, J., Roos, K., Dunkel, T., \& Harris, N. (2008). Nightmares and stress in children. Sleep and Hypnosis, 10(1), 19-25.

Schredl, M., \& Erlacher, D. (2004). Lucid dreaming frequency and personality. Personality and Individual Differences, 37(7), 1463-1473.

Schredl, M., Schafer, G., Hofmann, F., \& Jacob, S. (1999). Dream content and personality: Thick vs. Thin boundaries. Dreaming, 9(4), 257-263.

Selzer, M. L. (1971). The Michigan Alcoholism Screening Test: The quest for a new diagnostic instrument. The American Journal of Psychiatry, 127(12), 1653-1658.

Semiz, U. B., Basoglu, C., Ebrinc, S., \& Cetin, M. (2008). Nightmare disorder, dream anxiety, and subjective sleep quality in patients with borderline personality disorder. Psychiatry and Clinical Neurosciences, 62(1), 48-55.

Sjöström, N., Hetta, J., \& Wærn, M. (2009). Persistent nightmares are associated with repeat suicide attempt: A prospective study. Psychiatry Research, 170(2-3), 208-211.

Sjöström, N., Wærn, M., \& Hetta, J. (2007). Nightmares and sleep disturbances in relation to suicidality in suicide attempters. Sleep, 30(1), 91-95.

Spoormaker, V. I., \& van den Bout, J. (2006). Lucid dreaming treatment for nightmares: a pilot study. Psychotherapy And Psychosomatics, 75(6), 389-394.

St-Onge, M. l., Mercier, P., \& De Koninck, J. (2009). Imagery rehearsal therapy for frequent nightmares in children. Behavioral Sleep Medicine, 7(2), 81-98.

Talbot, M. (2009, November 16). Nightmare scenario, can we learn to rewrite our bad dreams. New Yorker, 43-51.

Tang, N. K. Y., Wright, K. J., \& Salkovskis, P. M. (2007). Prevalence and correlates of clinical insomnia co-occurring with chronic back pain. Journal Of Sleep Research, 16(1), 85-95. 
Tanskanen, A., Tuomilehto, J., ViinamÃaki, H., Vartiainen, E., Lehtonen, J., \& Puska, P. (2001). Nightmares as predictors of suicide. Sleep: Journal of Sleep and Sleep Disorders Research, 24(7), 844-847.

The Lucidity Institute. (1993). A Course in Lucid Dreaming. Palo Alto, CA: The Lucidity Institute.

Watson, D., Clark, L. A., \& Tellegen, A. (1988). Development and validation of brief measures of positive and negative affect: The PANAS scales. Journal of Personality and Social Psychology, 54(6), 1063-1070.

Weathers, F. W., Litz, B. T., Herman, D. S., Huska, J. A., \& Keane, T. M. (1993, October). The PTSD checklist (pcl); reliability, validity, and diagnostic utility. Paper presented at The International Society for Traumatic Stress Studies, San Antonio, TX.

Westermeyer, J., Yargic, I., \& Thuras, P. (2004). Michigan Assessment-Screening Test for Alcohol and Drugs (MAST/AD): Evaluation in a Clinical Sample. The American Journal on Addictions, 13(2), 151-162.

World Health Organization. (2007). International Statistical Classification of Diseases and Related Health Problems (10th ed.): World Health Organization. 
Appendix 1: The Beck Anxiety Inventory

The Beck Anxiety Inventory was not included in the appendix due to it being copyrighted. It may be purchased by Pearson Assessments. 


\section{Appendix 2: Beck Depression Inventory - II}

The Beck Depression Inventory - II was not included in the appendix due to it being copyrighted. It may be purchased by Pearson Assessments. 


\section{Appendix 3: Beck Hopelessness Scale}

The Beck Hopelessness Scale was not included in the appendix due to it being copyrighted. It may be purchased by Pearson Assessments. 
Appendix 4: Beck Scale for Suicide Ideation

The Beck Scale for Suicide Ideation was not included in the appendix due to it being copyrighted. It may be purchased by Pearson Assessments. 
Appendix 5: The Disturbing Dreams and Nightmares Severity Index

1. How often did you have disturbing dreams and/or nightmares: (Circle one, then follow the arrow)

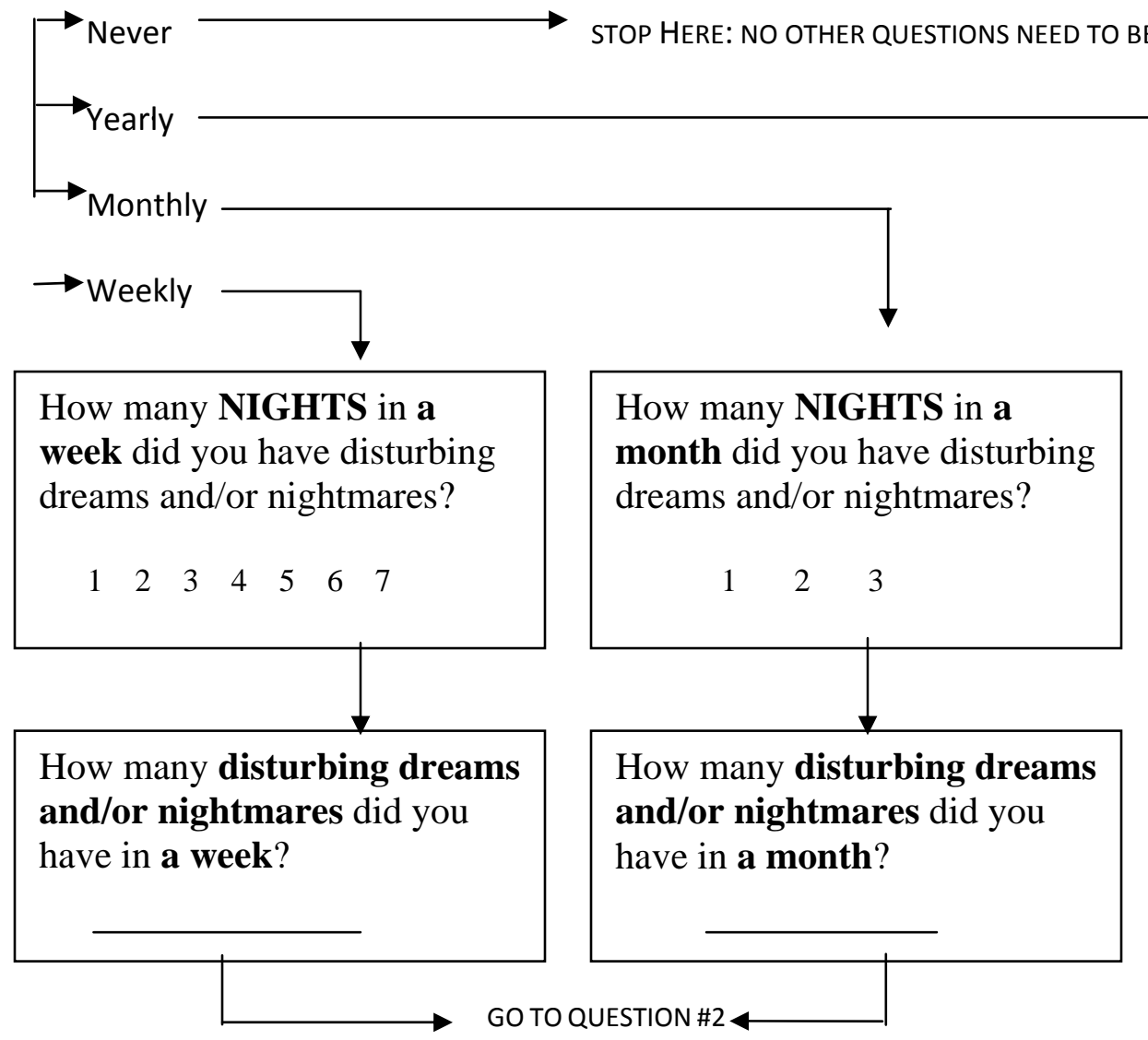

How many NIGHTS in a year did you have disturbing dreams and/or nightmares?

$\begin{array}{llllllllllll}1 & 2 & 3 & 4 & 5 & 6 & 7 & 8 & 9 & 10 & 11\end{array}$

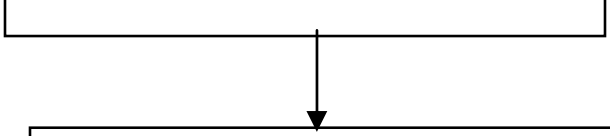

How many disturbing dreams and/or nightmares did you have in a year?

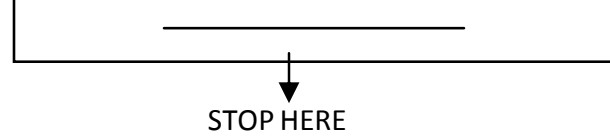

2. Please estimate the NUMBER of months or years you have had disturbing dreams and/or nightmares:

months years

3. On average, do your nightmares wake you up? (Circle answer)

$$
\text { Never/Rarely Occasionally Sometimes Frequently Always }
$$

4. How would you rate the SEVERITY of your disturbing dreams and/or nightmare problem? (Circle answer)

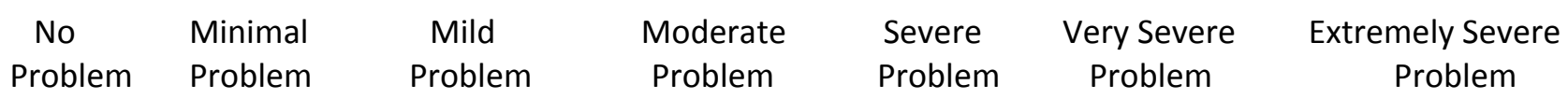

5. How would you rate the INTENSITY of your disturbing dreams and/or nightmares? (Circle answer)

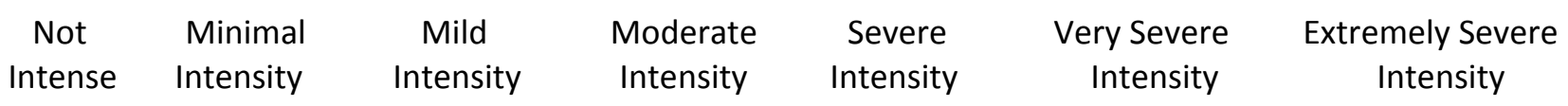


Appendix 6: The Insomnia Severity Index

Insomnia Severity Index (T. A. Nielsen et al.)

1. Please rate the current (i.e. last 2 weeks) SEVERITY of your insomnia problem(s).

Difficulty Falling

Asleep:

Difficulty Staying

Asleep:

Problem waking too

early:
None

0

0

0
Mild

1

1

1
Moderate

2

2

2
Severe

3

3

3

2. How SATISFIED/dissatisfied are you with your current sleep pattern?

Very Satisfied

Very Dissatisfied

$\begin{array}{lllll}0 & 1 & 2 & 3 & 4\end{array}$

3. To what extent do you consider your sleep problem to INTERFERE with your daily functioning (e.g. daytime fatigue, ability to function at work/daily chores, concentration, memory, mood, etc.)

\begin{tabular}{|c|c|c|c|c|}
\hline $\begin{array}{l}\text { Not at all } \\
\text { Interfering }\end{array}$ & A Little & Somewhat & Much & $\begin{array}{l}\text { Very Much } \\
\text { Interfering }\end{array}$ \\
\hline
\end{tabular}

0

1

2

3

4

4. How NOTICABLE to others do you think your sleeping problem is in terms of impairing the quality of your life?

Not at all

A Little Somewhat

Much

Very Much

Interfering

A Little Somewhat
Interfering

$$
0
$$

1

2

3

4

5. How WORRIED/distressed are you about your current sleep problem?

Not at all

0
A Little

1
Somewhat

2
Much

Very Much 
Appendix 7: The Michigan Assessment Screening Test for Alcohol and Drugs (MAST/AD)

\begin{tabular}{|c|c|c|c|}
\hline & & Yes & No \\
\hline 0 & Do you enjoy a drink or drug use every now and then? & 0 & 0 \\
\hline 1 & $\begin{array}{l}\text { Do you feel you are a normal drinker or drug user? (By normal, we } \\
\text { mean you drink or use drugs less than or as much as most other people) }\end{array}$ & 0 & 2 \\
\hline 2 & $\begin{array}{l}\text { Have you ever awakened the morning after some drinking or drug use } \\
\text { and found that you could not remember a part of the evening? }\end{array}$ & 2 & 0 \\
\hline 3 & $\begin{array}{l}\text { Does your wife, husband, a parent, or other near relative ever worry or } \\
\text { complain about your drinking or drug use? }\end{array}$ & 0 & 1 \\
\hline 4 & $\begin{array}{l}\text { Can you stop drinking or using drugs without a struggle after one or two } \\
\text { drinks or drug doses? }\end{array}$ & 0 & 2 \\
\hline 5 & Do you feel guilt about your drinking or drug use? & 1 & 0 \\
\hline 6 & Do friends or relatives think you are a normal drinker or drug user? & 0 & 2 \\
\hline 7 & Are you able to stop drinking or drug use when you want to? & 0 & 2 \\
\hline 8 & $\begin{array}{l}\text { Have you ever attended a meeting of Alcoholics Anonymous, Narcotics } \\
\text { Anonymous or other self-help group for drug use? }\end{array}$ & 5 & 0 \\
\hline 9 & Have you gotten into physical fights when drinking or using drugs? & 1 & 0 \\
\hline 10 & $\begin{array}{l}\text { Has your drinking or drug use ever created problems between you and } \\
\text { your wife, husband, a parent, or other relative? }\end{array}$ & 2 & 0 \\
\hline 11 & $\begin{array}{l}\text { Has your wife, husband (or other family members) ever gone to any one } \\
\text { for help about your drinking or drug use? }\end{array}$ & 2 & 0 \\
\hline 12 & Have you ever lost friends because of your drinking or drug use? & 2 & 0 \\
\hline 13 & $\begin{array}{l}\text { Have you ever gotten into trouble at work because of your drinking or } \\
\text { drug use? }\end{array}$ & 2 & 0 \\
\hline 14 & Have you ever lost a job because of drinking or drug use? & 2 & 0 \\
\hline 15 & $\begin{array}{l}\text { Have you ever neglected your obligations, your family, or your work for } \\
\text { two or more days in a row because you were drinking or using drugs? }\end{array}$ & 2 & 0 \\
\hline 16 & Do you drink or use drugs before noon fairly often & 1 & 0 \\
\hline 17 & Have you ever been told you have liver trouble? Cirrhosis? & 2 & 0 \\
\hline 18 & $\begin{array}{l}\text { After heavy drinking or drug use have you ever had Delirium Tremens } \\
\text { (D.T.'s) or severe shaking, or heard voices, seen things that weren't } \\
\text { really there? How many times? }\end{array}$ & 2 & 0 \\
\hline 19 & Have you ever gone to anyone for help about your drinking or drug use? & 5 & 0 \\
\hline 20 & Have you ever been in the hospital because of drinking or drug use? & 5 & 0 \\
\hline 21 & $\begin{array}{l}\text { Have you ever been a patient in a psychiatric hospital or on a psychiatric } \\
\text { ward of a general hospital where drinking or drug use was a part of the } \\
\text { problem that resulted in your hospitalization? }\end{array}$ & 2 & 0 \\
\hline 22 & $\begin{array}{l}\text { Have you ever been seen at a psychiatric or mental health clinic or gone } \\
\text { to any doctor, social worker, or clergyman for help because of any } \\
\text { emotional problem, where drinking or drug use was part of the } \\
\text { problem? }\end{array}$ & 2 & 0 \\
\hline 23 & $\begin{array}{l}\text { Have you ever been arrested for drunk driving, driving while } \\
\text { intoxicated, or driving under the influence of alcoholic beverages or } \\
\text { drugs? How many times? }\end{array}$ & 2 & 0 \\
\hline
\end{tabular}


24 Have you ever been arrested, or taken into custody, even for a few hours, because of other drunk or drug-related behavior? How many times? 
Appendix 8: The Positive and Negative Affect Schedule

This scale consists of a number of words that describe different feelings and emotions. Read each item and then mark the appropriate answer in the space next to that word. Indicate to what extent you have felt this way today. Use the following scale to record your answers.

1

Very slightly or not at all

interested

distressed

excited

upset

strong

guilty

scared

hostile

enthusiastic

proud
2

A little

3

Moderately

4

Quite a bit

5

Extremely
irritable
alert
ashamed
inspired
nervous
___ determined
attentive
__ jittery
active
afraid 


\section{Appendix 9: The PTSD Checklist Civilian Version}

INSTRUCTIONS TO PARTICIPANT: Below is a list of problems and complaints that people sometimes have in response to stressful experiences. Please read each one carefully, select the best option to indicate how much you have been bothered by that problem in the past month.

1. Repeated, disturbing memories, thoughts, or images of a stressful experience?

1. Not at all 2. A little bit 3. Moderately 4. Quite a bit 5. Extremely

2. Repeated, disturbing dreams of a stressful experience?

1. Not at all 2. A little bit 3. Moderately 4. Quite a bit 5. Extremely

3. Suddenly acting or feeling as if a stressful experience were happening again (as if you were reliving it)?

1. Not at all 2. A little bit 3. Moderately 4. Quite a bit 5. Extremely

4. Feeling very upset when something reminded you of a stressful experience?

1. Not at all 2. A little bit 3. Moderately 4. Quite a bit 5. Extremely

5. Having physical reactions (e.g., heart pounding, trouble breathing, sweating) when something reminded you of a stressful experience?

1. Not at all 2. A little bit 3. Moderately 4. Quite a bit 5. Extremely

6. Avoiding thinking about or talking about a stressful experience or avoiding having feelings related to it?

1. Not at all 2. A little bit 3. Moderately 4. Quite a bit 5. Extremely

7. Avoiding activities or situations because they reminded you of a stressful experience?

1. Not at all 2. A little bit 3. Moderately 4. Quite a bit 5. Extremely

8. Trouble remembering important parts of a stressful experience?

1. Not at all 2. A little bit 3. Moderately 4. Quite a bit 5. Extremely

9. Loss of interest in activities that you used to enjoy?

1. Not at all 2. A little bit 3. Moderately 4. Quite a bit 5. Extremely 
10. Feeling distant or cut off from other people?

1. Not at all 2. A little bit 3. Moderately 4. Quite a bit 5. Extremely

11. Feeling emotionally numb or being unable to have loving feelings for those close to you?

1. Not at all 2. A little bit 3. Moderately 4. Quite a bit 5. Extremely

12. Feeling as if your future will somehow be cut short?

1. Not at all 2. A little bit 3. Moderately 4. Quite a bit 5. Extremely

13. Trouble falling or staying asleep?

1. Not at all 2. A little bit 3. Moderately 4. Quite a bit 5. Extremely

14. Feeling irritable or having angry outbursts?

1. Not at all 2. A little bit 3. Moderately 4. Quite a bit 5. Extremely

15. Having difficulty concentrating?

1. Not at all 2. A little bit 3. Moderately 4. Quite a bit 5. Extremely

16. Being "super-alert" or watchful or on guard?

1. Not at all 2. A little bit 3. Moderately 4. Quite a bit 5. Extremely

17. Feeling jumpy or easily startled?

1. Not at all 2. A little bit 3. Moderately 4. Quite a bit 5. Extremely 


\section{Appendix 10: Qualitative Questions}

1. Please describe the nightmares you have most often.

2. Describe for me the effect that nightmares have had on your life.

3. Describe for me the things that might make you think you will have a nightmare.

4. Tell me about how you feel after having a nightmare. Can you describe for me how you feel physically? Psychologically? Emotionally?

5. Describe for me how your nightmares have changed over time.

6. Tell me about what you think may have contributed to your nightmares

7. Tell me about your discussions with health care providers about your nightmares?

8. When you think about your experience with nightmares, tell me about what seems most important to you. 


\section{Appendix 11: The Clinician Administered PTSD Scale}

The Clinician Administered PTSD Scale was not included in the appendix due to it being copyrighted. It may be purchased from Western Psychological Services. 


\section{Appendix 12: Suicide/Homicide Threat Procedures}

Participants will only be interviewed when a licensed psychologist is in the building and has agreed to be on-call for the project. In the unusual case that an individual might report current suicidal ideation at any point during the study, the experimenter will assess suicide risk. The experimenter will then remain with the participant and contact the psychologist who agreed to be on-call. Should he or she be unavailable, the experimenter will contact Dr. Fremouw, the clinic director. The experimenter will then consult with the psychologist who agreed to be oncall. Participants who have suicidal ideation but are not deemed to be at imminent risk of suicide will be given referrals to local mental health service providers for treatment. If a participant is at imminent risk of suicide, the experimenter and/or the consulting psychologist will call the Valley Health Care 24-hour crisis line, the Chestnut Ridge 24-hour help line, or Ruby Memorial Hospital to inform them of the situation and to develop a plan of action with the contacted agency. If the participant leaves against advice and it is determined that the participant is an imminent threat to himself/herself, or someone else, then the Sherriff's Department will be called to report the situation and develop a plan of action with the assistance of the representative contacted at the Sherriff's office. In the event that there is no answer at the Sherriff's Department, the experimenter or the consulting psychologist will call 911. 
Table 1: Exploratory Self-Report Measures

\begin{tabular}{|c|c|c|c|c|c|c|c|c|c|c|c|c|}
\hline & 001 & & & 008 & & & 010 & & & 012 & & \\
\hline & Base & Treat & Post & Base & Treat & Post & Base & Treat & Post & Base & Treat & Post \\
\hline DDNSI & 16 & 16 & 11.5 & 20 & 20 & 9.5 & 20 & 12 & 6 & 21 & 15 & 8.5 \\
\hline ISI & 13 & 7 & 2 & 14 & 8 & 8 & 14 & 10 & 9 & 9 & 8 & 6 \\
\hline BSS & 4 & 3 & 3 & 2 & 1 & 0 & 0 & 0 & 0 & 0 & 0 & 0 \\
\hline BDI & 22 & 13 & 7 & 12 & 15 & 10 & 2 & 3 & 3 & 11 & 17 & 8 \\
\hline BAI & 26 & 16 & 8 & 7 & 12 & 4 & 4 & 0 & 0 & 0 & 10 & 2 \\
\hline BHS & 1 & 2 & 1 & 2 & 1 & 1 & 2 & 1 & 3 & 1 & 1 & 0 \\
\hline PCL & 43 & 43 & 25 & 48 & 47 & 30 & 31 & 22 & 21 & 31 & 39 & 25 \\
\hline MAST/AD & 15 & 9 & 8 & 3 & 3 & 3 & 3 & 1 & 1 & 1 & 1 & 1 \\
\hline CAPS & 36 & N/A & 20 & 31 & N/A & 12 & 3 & N/A & 4 & 32 & N/A & 18 \\
\hline \multicolumn{13}{|c|}{ Note. ${ }^{*}$ Base $=$ start of baseline, Treat $=$ day of treatment, Post is four week follow-up. The } \\
\hline \multicolumn{13}{|c|}{ DDNSI = Disturbing Dreams and Nightmares Severity Index, the ISI = Insomnia Severity Index, } \\
\hline \multicolumn{13}{|c|}{ Beck Anxiety Inventory, the BHS = Beck Hopelessness Scale, The PCL = PTSD Checklist - } \\
\hline Civilian Veı & ion, & e MAS & T/AD & $=\mathrm{Mic}$ & $\operatorname{gan} A$ & essn & at $\mathrm{Sc}$ & ening & Test 1 & Alc & lan & Drugs, \\
\hline
\end{tabular}


Figure 1: Consort Diagram

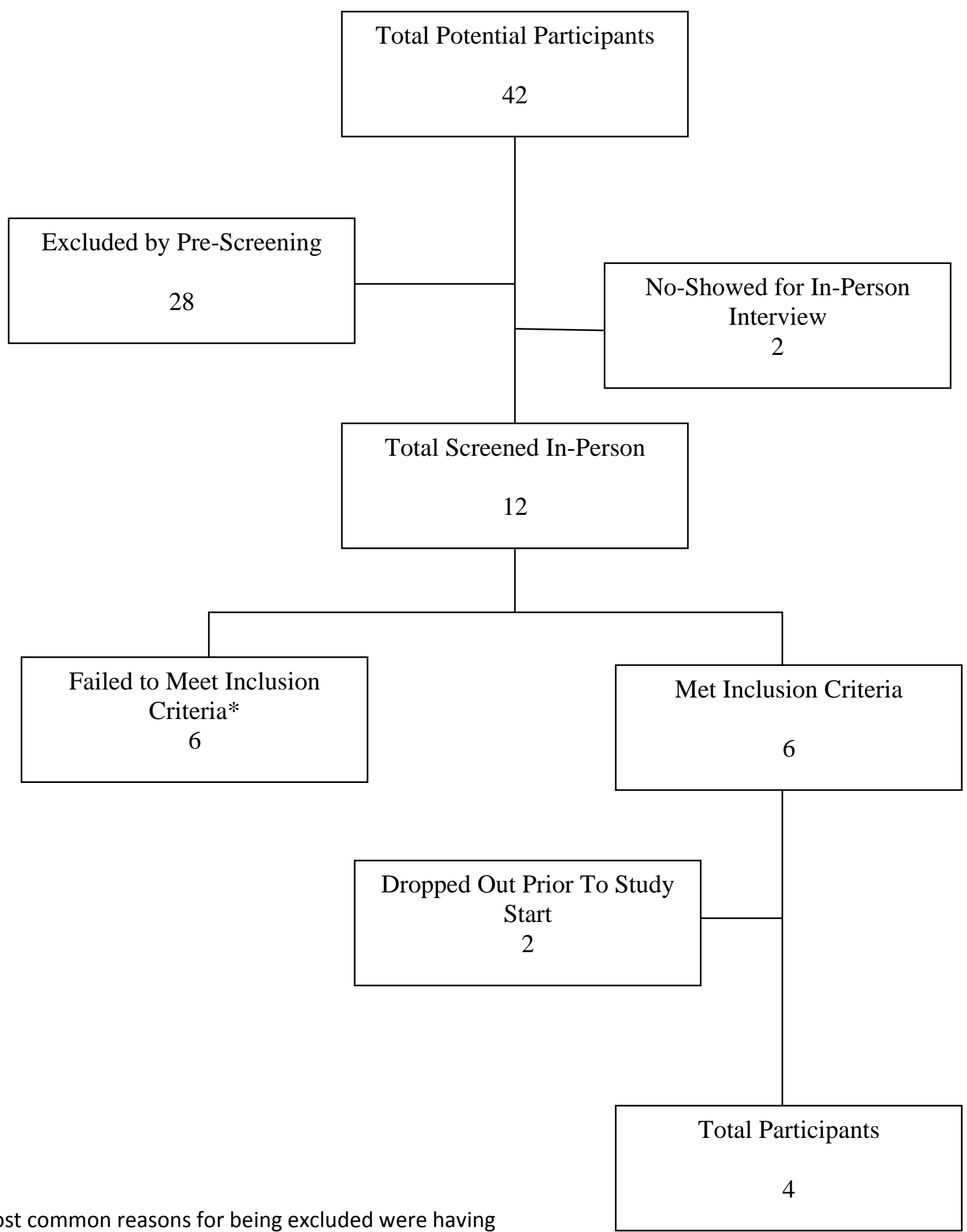

*Most common reasons for being excluded were having Low levels of suicidal ideation and not meeting criteria for nightmare disorder 
Figure 2: Nightmare Frequency Measured By Event Marker and Online Questionnaire

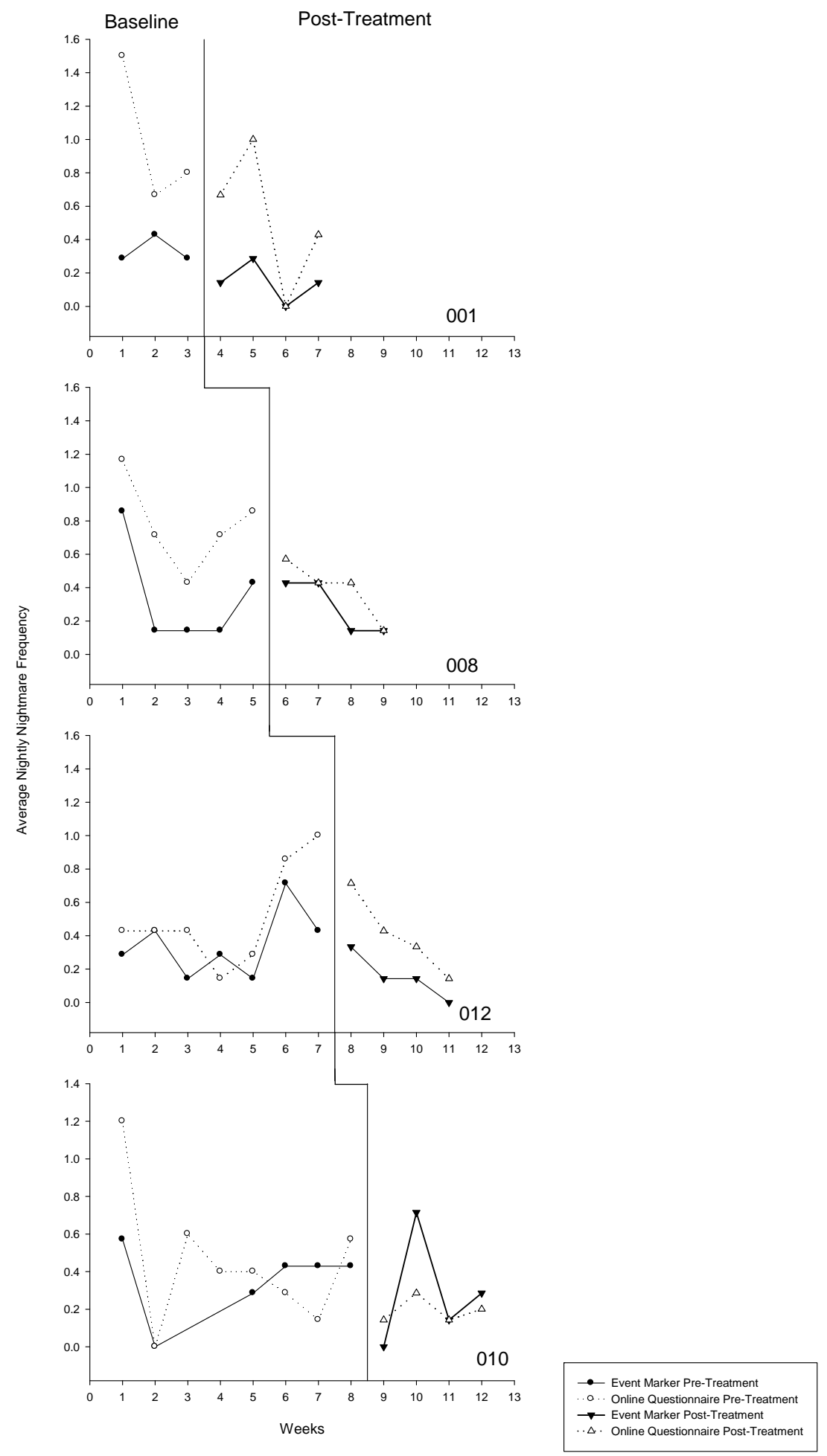


Figure 3: Average Nightmare Frequency (Event Marker)

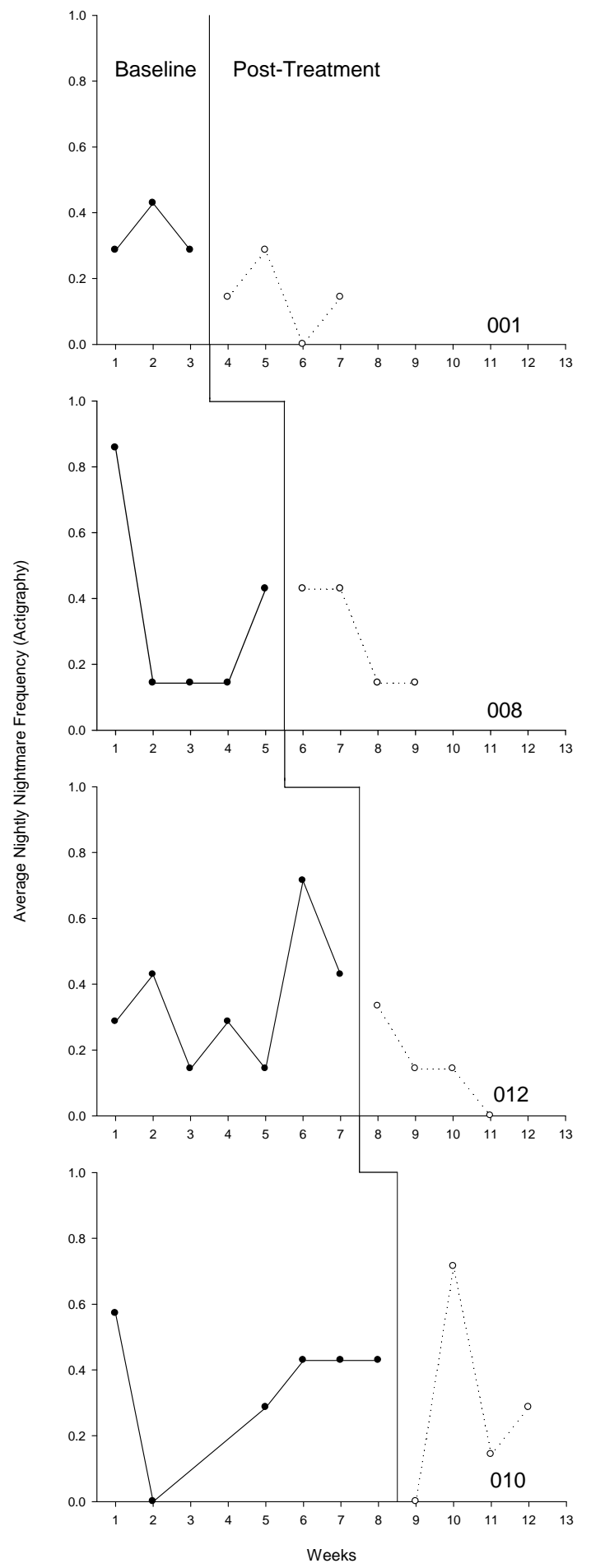


Figure 4: Average Nightmare Frequency (Online Questionnaire)
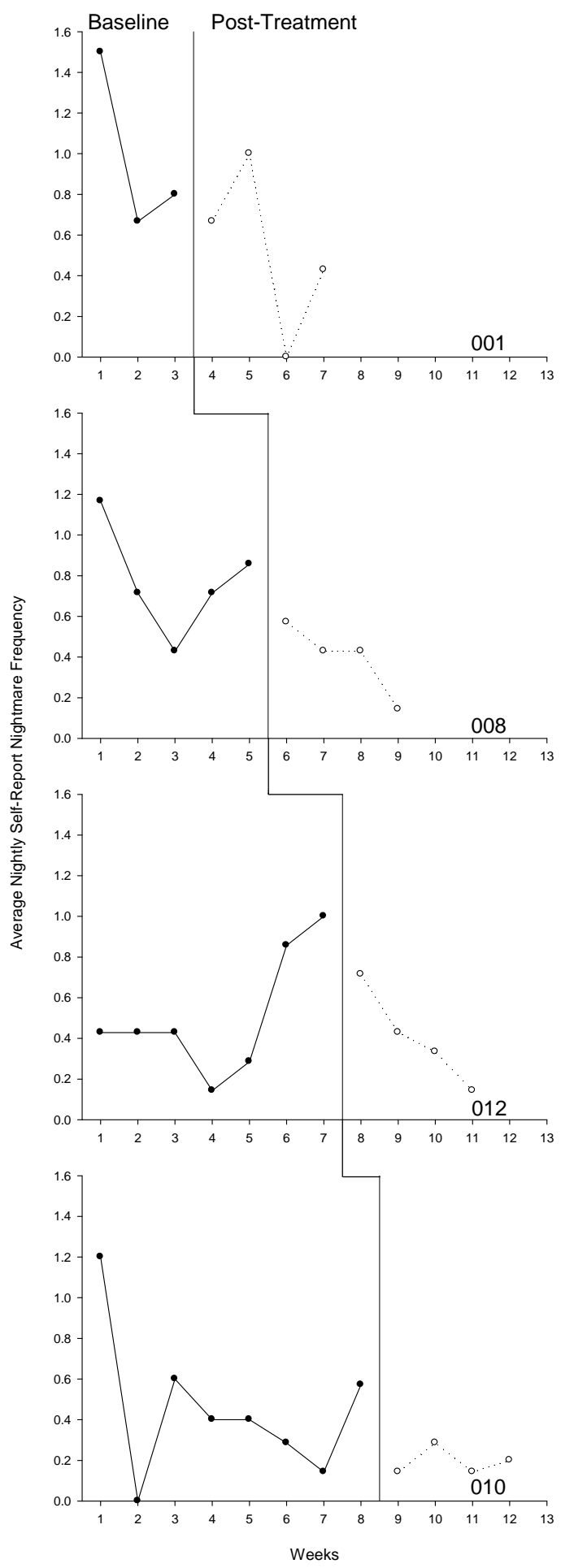
Figure 5: Average Nightmare Severity

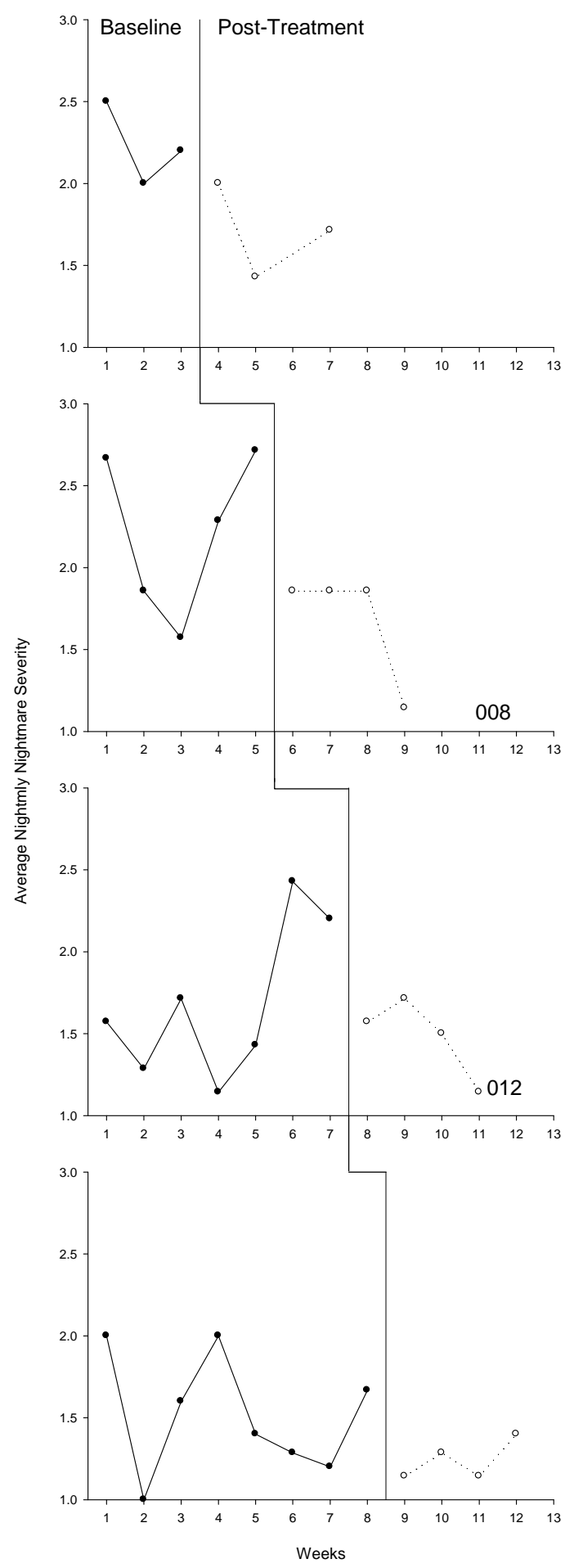


Figure 6: Average Nocturnal Awakenings

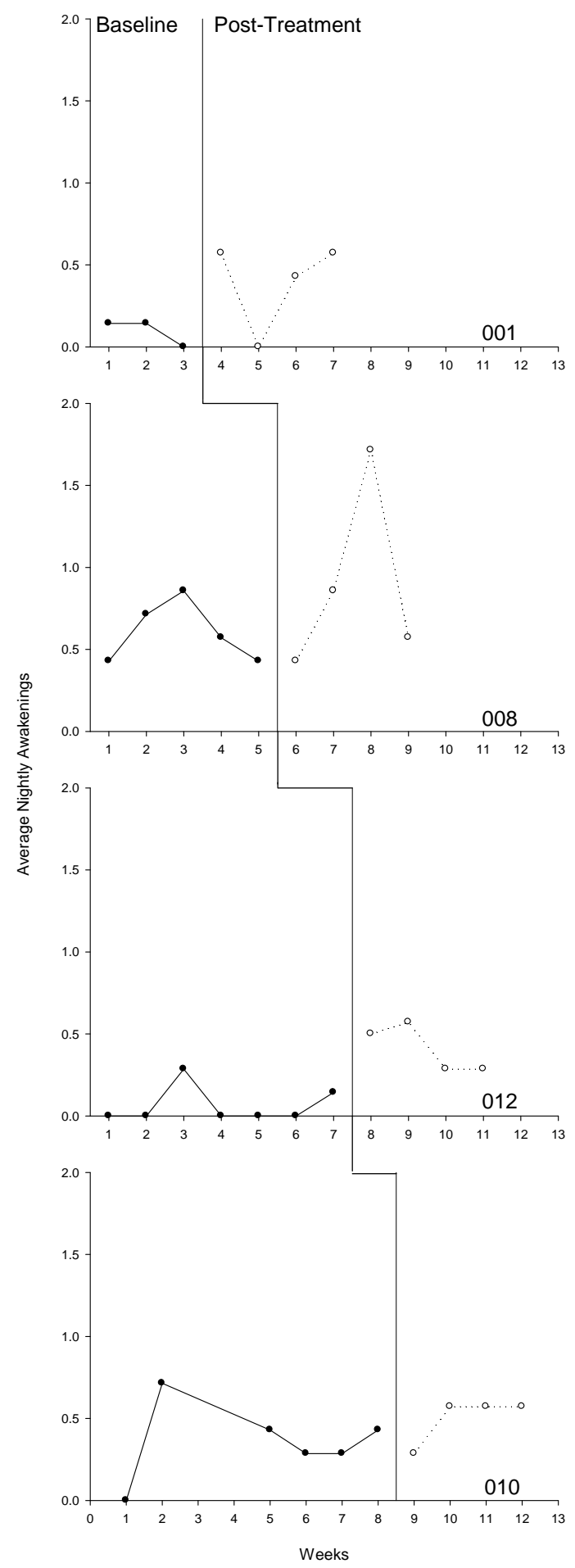


Figure 7: Average Sleep Efficiency

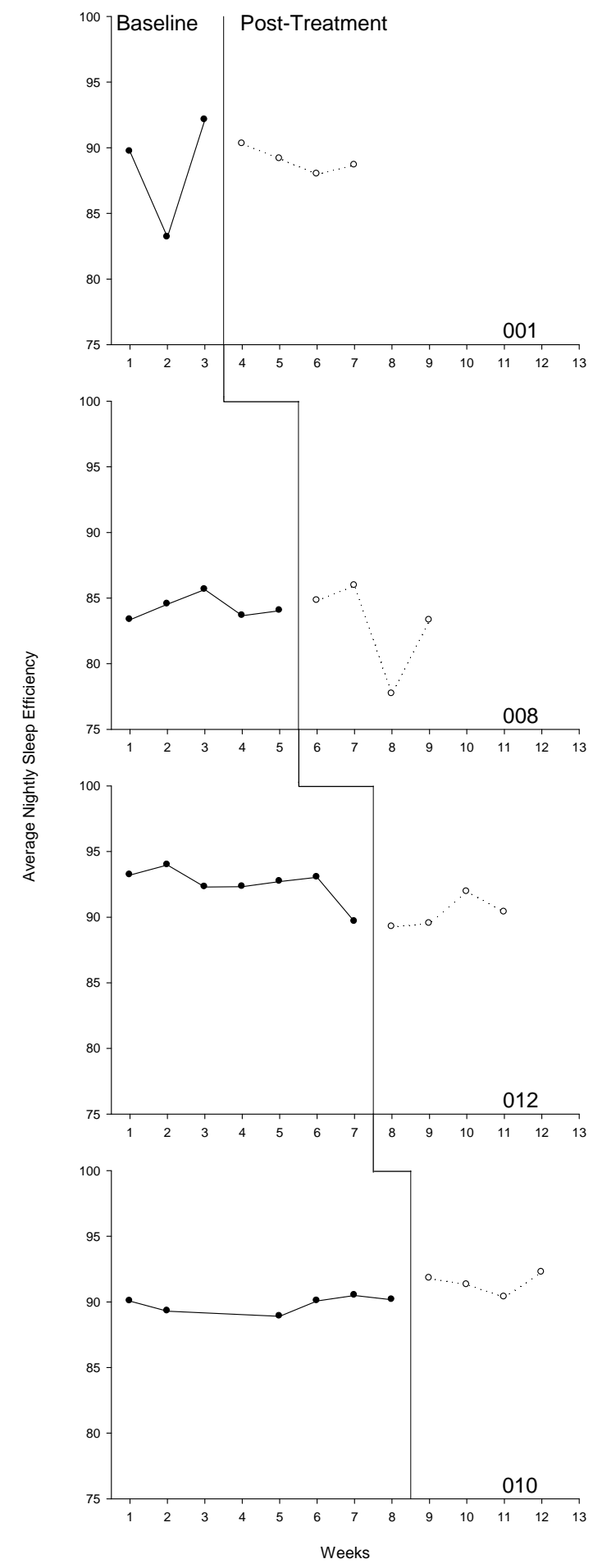


Figure 8: Average Negative Affect

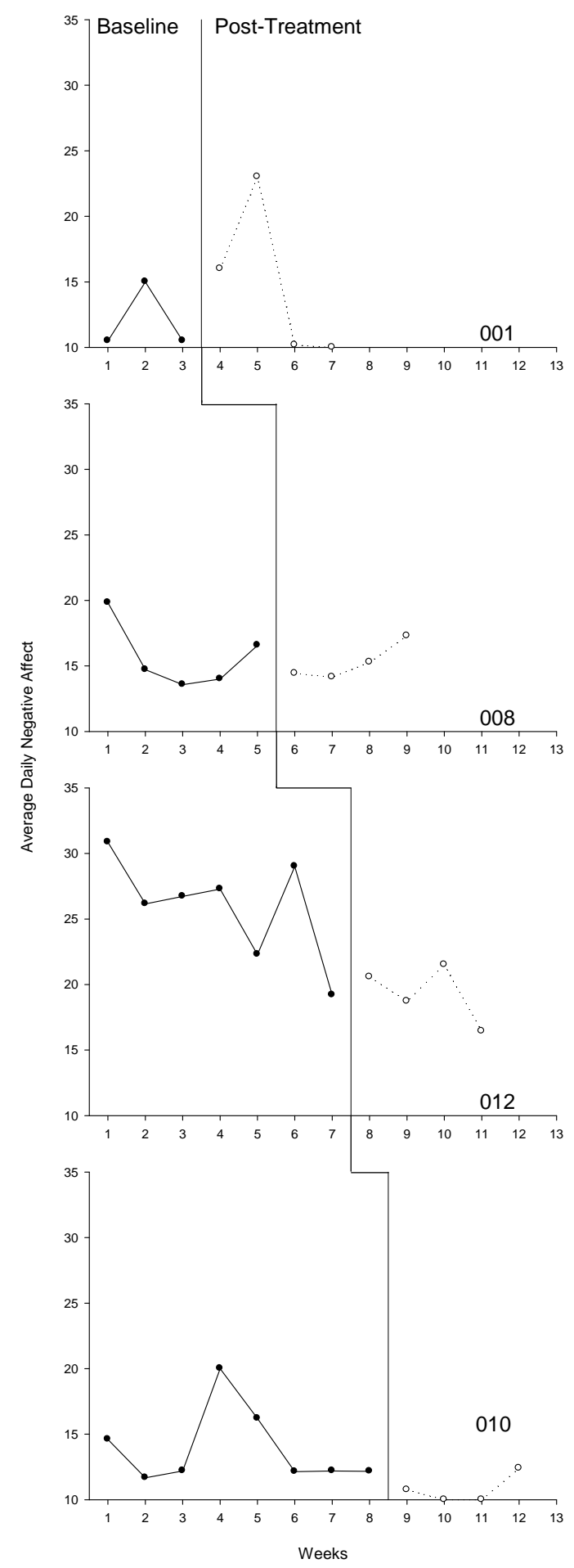

